\title{
FREE QUASICONFORMALITY IN BANACH SPACES II
}

\author{
Jussi Väisälä
}

\section{Introduction}

This paper is continuation to [V $\left.\ddot{a}_{4}\right]$. We consider homeomorphisms $f: G \rightarrow$ $G^{\prime}$ where $G$ and $G^{\prime}$ are domains in Banach spaces $E$ and $E^{\prime}$, respectively. In [Vä ${ }_{4}$ ] we introduced the class of freely $\varphi$-quasiconformal ( $\varphi$-FQC) maps, which in the case $E=R^{n}=E^{\prime}$ essentially agrees with the class of $K$-quasiconformal $(K$-QC) maps. We also considered some related concepts, in particular, $\varphi$-solid maps.

In this paper, the boundary and distortion properties of these maps are studied. In Section 2 we show that an isolated boundary point is removable for solid and FQC maps. Since quasihyperbolic geodesics do not always exist, we prove in Section 3 existence theorems for a generalized concept called a neargeodesic. Section 4 deals with a new tool called the coarse length of an arc. We also introduce the class of coarsely quasihyperbolic ( $\mathrm{CQH})$ maps, which includes all solid and hence all FQC maps. In Section 5 we relativize the theory of quasisymmetric (QS) and quasimöbius (QM) maps. The theory of uniform domains in Banach spaces is developed in Section 6. The theory of Sections 3-6 is applied in Section 7 to prove various results on maps of uniform domains. For example, a CQH map $f: G \rightarrow G^{\prime}$ between uniform domains extends to a homeomorphism $\bar{f}: \bar{G} \rightarrow \bar{G}^{\prime}$, which is QM rel $\partial G$. In particular, the induced boundary map $f_{0}: \partial G \rightarrow \partial G^{\prime}$ is QM. If $f$ is FQC, then $f$ itself is QM. Many of the results are also new in the classical case $E=R^{n}=E^{\prime}$. In Section 8 we apply the idea of relative quasisymmetry to reprove and generalize the recent interesting distortion theorem of $\mathrm{D}$. Cooper on $\mathrm{CQH}$ maps of the $n$-ball.

We shall use the terminology and notation of [Vä 4 ]. In particular, $X$ and $Y$ will be metric spaces, $E$ and $E^{\prime}$ will be real Banach spaces and $G \subset E, G^{\prime} \subset E^{\prime}$ domains. In the present paper we shall also assume that $\operatorname{dim} E \geq 2, \operatorname{dim} E^{\prime} \geq 2$. The closure $\bar{A}$ and the boundary $\partial A$ of a set $A \subset E$ are taken in the extended space $\dot{E}=E \cup\{\infty\}$. References to [V $\left.\ddot{\mathrm{a}}_{4}\right]$ will be given in the form I.2.5, which means the result 2.5 of $\left[\mathrm{V}_{4}\right]$.

I thank P. Alestalo, J. Luukkainen and O. Martio for valuable remarks.

AMS classification: Primary 30C60, secondary 46B20. 


\section{Isolated boundary points}

2.1. Introduction to Section 2. Suppose that $x_{0}$ is an isolated boundary point of a domain $G \subset E$ and that $f: G \rightarrow G^{\prime}$ is a homeomorphism. If $E=R^{n}=E^{\prime}$ and $f$ is $K-\mathrm{QC}$, it is well known that $f$ has a $K$-QC extension to $G \cup\left\{x_{0}\right\}$. We shall prove the corresponding result for solid and FQC maps.

For solid maps the result is new also in $R^{n}$. However, the proof for this case would be considerably shorter. For example, we know that the cluster set $\operatorname{clus}\left(f, x_{0}\right)$ is a component $C$ of $\partial G^{\prime}$. For small $r$ the QH diameter of $S\left(x_{0}, r\right)$ is $\pi$. If $C$ contains more than one point, then the QH diameter of $f S\left(x_{0}, r\right)$ tends to $\infty$ as $r \rightarrow 0$, which contradicts the solidity of $f$. This proves that $f$ has a limit at $x_{0}$. In the infinite-dimensional case we can say very little of the cluster set by topological reasons, which makes the proof much longer.

We start with a basic result of Banach geometry.

2.2. Lemma. Let $S=S\left(x_{0}, r\right)$ be a sphere in $E$. Then each pair of points in $S$ can be joined by a 2 -quasiconvex arc in $S$.

Proof. Let $a, b \in S, a \neq b$. Let $T$ be a 2-dimensional linear subspace of $E$ containing $a$ and $b$. Then $T \cap S$ is a topological circle, and the points $a, b$ divide $T \cap S$ to arcs $\gamma_{1}, \gamma_{2}$. Assuming $l\left(\gamma_{1}\right) \leq l\left(\gamma_{2}\right)$, the arc $\gamma_{1}$ is 2-quasiconvex by [Sc, 4.4]. ㅁ

2.3. Remark. In a Hilbert space we can replace the constant 2 of 2.2 by $\pi / 2$. The bound 2 is sharp in the plane with the norm $|x|=\left|x_{1}\right|+\left|x_{2}\right|$.

2.4. Notation. For a set $A \subset G$ we let $k(A)$ denote the QH diameter of $A$, and $k(A, B)$ is the $\mathrm{QH}$ distance between two nonempty sets $A, B \subset G$. In $G^{\prime}$ we replace $k$ by $k^{\prime}$.

2.5. Lemma. Suppose that $x_{0}$ is a finite isolated boundary point of $G$ and that $B\left(x_{0}, 2 r\right) \subset G \cup\left\{x_{0}\right\}$. Suppose also that $a, b \in G$ with $\left|a-x_{0}\right| \leq\left|b-x_{0}\right| \leq r$. Then

$$
k(a, b) \leq \ln \frac{\left|b-x_{0}\right|}{\left|a-x_{0}\right|}+4,
$$

and thus $k\left(S\left(x_{0}, r\right)\right) \leq 4$.

Proof. We may assume that $x_{0}=0$. Set $y=|b| a /|a|$. By 2.2 we can choose an arc $\alpha \subset S\left(x_{0},|b|\right)$ joining $y$ and $b$ with $l(\alpha) \leq 2|b-y| \leq 4|b|$. Then $\gamma=[a, y] \cup \alpha$ joins $a$ and $b$ in $G$, and hence

$$
k(a, b) \leq l_{k}(\gamma)=\int_{|a|}^{|b|} \frac{d t}{t}+\frac{l(\alpha)}{|b|} \leq \ln \frac{|b|}{|a|}+4 . \text { 口 }
$$

2.6. Lemma. Suppose that $G \neq E, G^{\prime} \neq E^{\prime}$ and that $f: G \rightarrow G^{\prime}$ is solid. Let $A \subset G$ with $k(A)<\infty$. Then $f A$ is bounded, $d\left(f A, \partial G^{\prime}\right)>0, \overline{f A} \subset G^{\prime}$ and $\partial f A=f \partial A$. 
Proof. By solidity we have $k^{\prime}(f A)<\infty$. By I.2.2(1) this implies that $f A$ is bounded and that $d\left(f A, \partial G^{\prime}\right)>0$. Hence $\overline{f A} \subset G^{\prime}$, and $\partial f A$ is the boundary of $f A$ in the topology of $G^{\prime}$. Since $f$ is a homeomorphism, we have $\partial f A=f \partial A$. व

2.7. Theorem. Suppose that $x_{0}$ is an isolated boundary point of $G \neq E$ and that $f: G \rightarrow G^{\prime}$ is $\varphi$-solid. Then $f$ has a limit $y_{0} \in \dot{E}^{\prime}$ at $x_{0}$, and $y_{0}$ is an isolated boundary point of $\partial G^{\prime}$. Setting

$$
G_{1}=G \cup\left\{x_{0}\right\}, \quad G_{1}^{\prime}=G^{\prime} \cup\left\{y_{0}\right\}, \quad f_{1}\left(x_{0}\right)=y_{0},
$$

we obtain an extension of $f$ to a homeomorphism $f_{1}: G_{1} \rightarrow G_{1}^{\prime}$. If $x_{0} \neq \infty$, $y_{0} \neq \infty$ and $G_{1} \neq E$, then $f_{1}$ is $\varphi_{1}$-solid with $\varphi_{1}=\varphi_{1}(\varphi)$.

Proof. Performing a preliminary inversion if necessary, we may assume that $x_{0} \neq \infty$. By a translation we can normalize $x_{0}=0$. We break the proof to three lemmas $2.8,2.15$ and 2.16 .

2.8. Lemma. The map $f$ has a limit $y_{0}$ at 0 , possibly $y_{0}=\infty$.

Proof. Writing $U(r)=B(r) \backslash\{0\}$ we choose $r_{0}>0$ with $U\left(2 r_{0}\right) \subset G$. We may assume that $0 \in f S\left(r_{0}\right)$. If $f x \rightarrow \infty$ as $x \rightarrow x_{0}$, there is nothing to prove. We may therefore assume that there are $R>0$ and a sequence of points $x_{j} \in U\left(r_{0}\right)$ such that $r_{j}=\left|x_{j}\right| \rightarrow 0$ as $j \rightarrow \infty$, the sequence $\left(r_{j}\right)$ is strictly decreasing, and $\left|f x_{j}\right| \leq R$ for all $j$. Since $E^{\prime}$ is complete, it suffices to show that $d\left(f U\left(r_{j}\right)\right) \rightarrow 0$ as $j \rightarrow \infty$. The proof consists of six steps.

Step 1. Writing $S_{j}=S\left(r_{j}\right)$ for $j \geq 0$ we infer from I.2.2(1) that

$$
k\left(S_{j}, S_{0}\right) \geq \ln \frac{r_{0}}{r_{j}} \rightarrow \infty
$$

as $j \rightarrow \infty$. By solidity this implies

$$
\lim _{j \rightarrow \infty} k^{\prime}\left(f S_{j}, f S_{0}\right)=\infty
$$

If $B(2 R) \subset G^{\prime}$, I.2.5 implies $k^{\prime}\left(f x_{j}, 0\right) \leq 1$ for all $j$. By (2.9) this is impossible, and hence

$$
B(2 R) \cap \partial G^{\prime} \neq \emptyset
$$

Step 2. For $j \geq 1$ let $A_{j}$ be the annulus $B\left(r_{0}\right) \backslash \bar{B}\left(r_{j}\right)$. We next show that there is $R_{1}=R_{1}(R, \varphi)>0$ such that

$$
f A_{j} \subset \bar{B}\left(R_{1}\right)
$$


for all $j \geq 1$. If $x \in S_{j}$, then I.2.2(1), 2.5 and (2.10) imply

$$
\left|f x-f x_{j}\right| \leq \delta^{\prime}\left(f x_{j}\right) e^{k^{\prime}\left(f x, f x_{j}\right)} \leq 3 R e^{\varphi(4)} .
$$

Hence $f S_{j} \subset \bar{B}\left(R_{1}\right)$ with $R_{1}=3 R e^{\varphi(4)}+R$. Since $0 \in f S_{0}$, the same argument shows that $f S_{0} \subset \bar{B}\left(R_{1}\right)$. From 2.5 we infer

$$
k\left(\bar{A}_{j}\right) \leq \ln \frac{r_{0}}{r_{j}}+4<\infty .
$$

By 2.6 this implies

$$
\partial f A_{j}=f S_{0} \cup f S_{j} .
$$

Hence $f A_{j} \subset \bar{B}\left(R_{1}\right)$, since otherwise $\dot{E} \backslash \bar{B}\left(R_{1}\right)$ would be a connected set meeting $f A_{j}$ and $\dot{E} \backslash f A_{j}$ but not $\partial f A_{j}$.

Step 3. We show that $\alpha_{j}=d\left(f S_{j}, f S_{0}\right)>0$ for $j \geq 1$. By (2.12) and 2.6 we have $d\left(f \bar{A}_{j}, \partial G^{\prime}\right)=q_{j}>0$. If $x \in S_{0}, y \in S_{j}$ and $|f x-f y| \leq q_{j} / 2$, then I.2.5 yields

$$
k^{\prime}(f x, f y) \leq \frac{2|f x-f y|}{\delta^{\prime}(f x)} \leq \frac{2|f x-f y|}{q_{j}} .
$$

On the other hand, I.2.2(1) gives $k(x, y) \geq \ln \left(r_{0} / r_{j}\right)$. Hence

$$
|f x-f y| \geq \frac{q_{j}}{2} \varphi^{-1}\left(\ln \frac{r_{0}}{r_{j}}\right)=\beta_{j}>0,
$$

which implies $\alpha_{j} \geq \beta_{j} \wedge\left(q_{j} / 2\right)>0$.

Step 4 . We show that $f S_{j}$ and $f S_{0}$ can be joined by an $\operatorname{arc} \gamma_{j} \subset f \bar{A}_{j}$ which consists of two line segments. Since $A_{j}$ is connected, there is $z \in A_{j}$ such that

$$
d\left(f z, f S_{0}\right)=d\left(f z, f S_{j}\right)=\lambda .
$$

Choose points $a_{0} \in f S_{0}$ and $a_{j} \in f S_{j}$ such that

$$
\left|f z-a_{0}\right| \wedge\left|f z-a_{j}\right| \leq \lambda+\alpha_{j} / 2
$$

Replacing $a_{0}$ by a point in $\left[f z, a_{0}\right]$ we may assume that $\left[f z, a_{0}\right) \cap f S_{0}=\emptyset$, and similarly $\left[f z, a_{j}\right) \cap f S_{j}=\emptyset$. If there is a point $u \in\left[f z, a_{0}\right] \cap f S_{j}$, then

$$
|u-f z| \geq d\left(f z, f S_{j}\right)=\lambda,
$$

and hence $\left|u-a_{0}\right| \leq \alpha_{j} / 2$. Since $\alpha_{j}=d\left(f S_{j}, f S_{0}\right) \leq\left|u-a_{0}\right|$, we obtain a contradiction. Thus $\left[f z, a_{0}\right] \cap f S_{j}=\emptyset$. Similarly $\left[f z, a_{j}\right] \cap f S_{0}=\emptyset$. By $(2.13)$ the arc $\gamma_{j}=\left[f z, a_{0}\right] \cup\left[f z, a_{j}\right]$ lies in $\bar{A}_{j}$. 
Step 5. We next show that

$$
\lim _{j \rightarrow \infty} d\left(f S_{j}\right)=0 .
$$

Set $\delta_{j}=d\left(\gamma_{j}, \delta G^{\prime}\right)$. By Step 4 and (2.11) we obtain

$$
k^{\prime}\left(f S_{0}, f S_{j}\right) \leq l_{k}\left(\gamma_{j}\right) \leq l\left(\gamma_{j}\right) / \delta_{j} \leq 4 R_{1} / \delta_{j} .
$$

By (2.9) this implies that $\delta_{j} \rightarrow 0$ as $j \rightarrow \infty$. Hence $d\left(\partial f A_{j}, \partial G^{\prime}\right) \rightarrow 0$. In Step 3 we observed that $d\left(f S_{0}, \partial G^{\prime}\right)>0$. In view of (2.13) we obtain $d\left(f S_{j}, \partial G^{\prime}\right) \rightarrow 0$. On the other hand, we have $k^{\prime}\left(f S_{j}\right) \leq \varphi(4)$, and hence by I.2.2(1),

$$
d\left(f S_{j}\right) \leq 2 d\left(f S_{j}, \partial G^{\prime}\right) e^{\varphi(4)},
$$

which gives (2.14).

Step 6. As the final step we prove the original claim $d\left(f U\left(r_{j}\right)\right) \rightarrow 0$. Let $\varepsilon>0$. By (2.14) there is $j$ such that $d\left(f S_{i}\right) \leq \varepsilon$ for all $i \geq j$. It suffices to show that $d\left(f U\left(r_{j}\right)\right) \leq 4 \varepsilon$. Let $A_{i j}$ be the annulus $B\left(r_{j}\right) \backslash \bar{B}\left(r_{i}\right)$ in $G$. It suffices to show that $d\left(f A_{i j}\right) \leq 4 \varepsilon$ for all $i>j$. Choose closed balls $B_{i}$ and $B_{j}$ of radius $\varepsilon$ containing $f S_{i}$ and $f S_{j}$, respectively. If $B_{i} \cap B_{j}=\emptyset$, then $\dot{E}^{\prime} \backslash\left(B_{1} \cup B_{2}\right)$ is a connected set meeting $\dot{E}^{\prime} \backslash f A_{i j}$. As in (2.13) we obtain $\partial f A_{i j}=f S_{i} \cup f S_{j} \subset B_{1} \cup B_{2}$. Hence $f A_{i j} \subset B_{1} \cup B_{2}$. Since $f A_{i j}$ is connected, this is impossible. Consequently, $B_{i}$ meets $B_{j}$, and hence $\partial f A_{i j}$ is contained in a ball $B$ of radius $2 \varepsilon$. This implies that $f A_{i j} \subset B$, and hence $d\left(f A_{i j}\right) \leq 4 \varepsilon$. Lemma 2.8 is proved. ㅁ

2.15. Lemma. Set $G_{1}=G \cup\{0\}, G_{1}^{\prime}=G^{\prime} \cup\left\{y_{0}\right\}$. Then $G_{1}$ and $G_{1}^{\prime}$ are domains in $E$ and $\dot{E}^{\prime}$, respectively. The extension $f_{1}: G_{1} \rightarrow G_{1}^{\prime}$ with $f_{1}(0)=y_{0}$ is a homeomorphism.

Proof. Clearly $y_{0} \in \partial G^{\prime}$, and $G_{1}$ is a domain. Performing a preliminary inversion and a translation, if necessary, we may assume that $y_{0}=0$. Write again $U(r)=B(r) \backslash\{0\}$ and choose $r_{0}>0$ with $U\left(2 r_{0}\right) \subset G$. Since $k\left(S\left(r_{0}\right)\right) \leq 4<\infty$ by 2.5 , Lemma 2.6 implies that $d\left(f S\left(r_{0}\right), 0\right)=t_{0}>0$. We show that $B\left(t_{0}\right) \cap \partial G^{\prime}=$ $\{0\}$. Assume that this is false. Since $f U\left(r_{0}\right)$ is an open set meeting $B\left(t_{0}\right)$, we can choose points $z$ and $z_{1}$ in $B\left(t_{0}\right)$ such that $z \in f U\left(r_{0}\right), z_{1} \in \partial G^{\prime}$, and $0 \notin\left[z, z_{1}\right]$. Replacing $z_{1}$ by a point in $\left[z, z_{1}\right]$ we may assume that $\beta=\left[z, z_{1}\right) \subset G^{\prime}$. Then $\alpha=f^{-1} \beta$ does not meet $S\left(r_{0}\right)$, and hence $\alpha \subset B\left(r_{0}\right)$. Since $f_{1}$ is continuous at $0, \bar{\alpha}$ does not contain 0 . Hence $k(\alpha)<\infty$, which implies $k^{\prime}(\beta)<\infty$. This is a contradiction, since $\bar{\beta}$ meets $\partial G^{\prime}$.

We have proved that 0 is an isolated boundary point of $G^{\prime}$. This implies that $G_{1}^{\prime}$ is a domain. Moreover, $f_{1}: G_{1} \rightarrow G_{1}^{\prime}$ is a continuous bijection. It remains to show that $f_{1}^{-1}$ is continuous at the origin.

Let $0<r<r_{0}$. As above, $d(f S(r), 0)=t>0$. Then $f^{-1} U(t)$ is a connected set meeting $B(r)$ but not $S(r)$. Hence $f^{-1} U(t) \subset B(r)$, which implies the continuity of $f_{1}^{-1}$ at 0 . $\square$ 
2.16. Lemma. Suppose that $G_{1} \neq E$ and $y_{0} \neq \infty$. Then $G_{1}^{\prime} \neq E^{\prime}$ and $f_{1}: G_{1} \rightarrow G_{1}^{\prime}$ is $\varphi_{1}$-solid with $\varphi_{1}=\varphi_{1}(\varphi)$.

Proof. We may again assume that $y_{0}=0$. Assume that $G_{1}^{\prime}=E^{\prime}$. This means that $G^{\prime}=E^{\prime} \backslash\{0\}$. By Lemma $2.8, f_{1}^{-1}$ has a limit $x_{1} \neq 0$ at $\infty$, possibly $x_{1}=\infty$. Since $G_{1} \neq E$, we can choose neighborhoods $U$ of 0 and $V$ of $x_{1}$ such that $\bar{U} \cap \bar{V}=\emptyset$ and $\dot{E} \backslash(U \cup V)$ meets $\partial G$. Then for the set $A=G \backslash(U \cup V)$ we have $k(A)=\infty$. However, $\overline{f A}$ does not meet $\{0, \infty\}$, and hence $k^{\prime}(f A)<\infty$. This contradiction proves that $G_{1}^{\prime} \neq E^{\prime}$.

By auxiliary similarities we can normalize the situation so that $d\left(0, \partial G_{1}\right)=$ $1=d\left(0, \partial G_{1}^{\prime}\right)$. We first show that there are a number $r_{0}=r_{0}(\varphi), 0<r_{0}<1 / 2$, and an increasing homeomorphism $\psi=\psi_{\varphi}:\left[0, r_{0}\right] \rightarrow[0,1 / 2]$ such that

$$
\left|f_{1} x\right| \leq \psi(|x|)
$$

whenever $|x| \leq r_{0}$.

We first observe that for each $r \in(0,1)$ we have $\overline{f_{1} B(r)} \subset G_{1}^{\prime}$ and hence $\partial f_{1} B(r)=f S(r)$. Indeed, there is $r_{1}<r$ with $f_{1} B\left(r_{1}\right) \subset B(1 / 2)$. The annulus $A=B(r) \backslash B\left(r_{1}\right)$ has a finite $\mathrm{QH}$ diameter in $G$. By 2.6 , this implies $\overline{f A} \subset G^{\prime}$, and hence $f_{1} B(r) \subset G_{1}^{\prime}$.

Set $\varepsilon=\varepsilon(\varphi)=e^{-\varphi(4)} / 2$, and choose a point $z \in \partial G_{1}^{\prime}$ with $|z| \leq 1+\varepsilon$. Let $\beta$ be the ray from $z$ through 0 . We can choose points $y_{1}, y_{2} \in \beta \cap f S(1 / 2)$ such that $0 \in\left[y_{1}, y_{2}\right] \subset f_{1} \bar{B}(1 / 2)$ and $y_{1} \in[z, 0]$. Writing $x_{j}=f^{-1} y_{j}$ we have $k\left(x_{1}, x_{2}\right) \leq 4$ by 2.5 . Setting $q=\left|y_{1}-z\right|$ and assuming $q \leq 1 / 2$ we have

$$
d\left(y_{1}, \partial G^{\prime}\right) \leq q, \quad\left|y_{1}-y_{2}\right| \geq\left|y_{1}\right| \geq 1-q .
$$

By I.2.2(1) these yield

$$
k^{\prime}\left(y_{1}, y_{2}\right) \geq \ln \frac{1}{q} .
$$

Since $f$ is $\varphi$-solid, we obtain

$$
q \geq e^{-\varphi(4)}=2 \varepsilon,
$$

which is also valid if $q \geq 1 / 2$.

Next assume that $0<r<1 / 2$. Choose a point $x_{3} \in S(r)$ such that $f x_{3}=$ $y_{3} \in\left[y_{1}, 0\right]$. If $y \in\left[y_{1}, y_{3}\right]$ and $z_{1} \in \partial G_{1}$, we have by (2.18)

$$
1 \leq\left|z_{1}\right| \leq|y|+\left|y-z_{1}\right| \leq 1+\varepsilon-q+\left|y-z_{1}\right| \leq 1-\varepsilon+\left|y-z_{1}\right|
$$

which implies

$$
d\left(y, \partial G^{\prime}\right) \geq \varepsilon \wedge\left|y_{3}\right|=t
$$




\section{Hence}

$$
k^{\prime}\left(y_{1}, y_{3}\right) \leq\left|y_{1}-y_{3}\right| / t<2 / t
$$

On the other hand, $k\left(x_{3}, x_{1}\right) \geq \ln (1 / 2 r)$, and hence

$$
t<\frac{2}{\varphi^{-1}(\ln (1 / 2 r))}=\psi_{0}(r) \rightarrow 0
$$

as $r \rightarrow 0$. Setting $r_{0}=e^{-\varphi(2 / \varepsilon)} / 2=r_{0}(\varphi)$ we have $\psi_{0}\left(r_{0}\right)=\varepsilon$. Hence

$$
\left|y_{3}\right| \leq \psi_{0}(r)
$$

for $0<r \leq r_{0}$.

Let $x \in S(r)$. Then $|f x| \leq\left|y_{3}\right|$ or

$$
k^{\prime}\left(f x, y_{3}\right) \geq \ln \left(1+\frac{\left|f x-y_{3}\right|}{\left|y_{3}\right|}\right) \geq \ln \frac{|f x|}{\left|y_{3}\right|} .
$$

Since $k\left(x, x_{3}\right) \leq 4$, this and (2.19) yield

$$
|f x| \leq e^{\varphi(4)}\left|y_{3}\right| \leq e^{\varphi(4)} \psi_{0}(r)=\psi(r)
$$

for $0<r \leq r_{0}$. Setting $\psi(0)=0$ we obtain (2.17). Moreover, $\psi\left(r_{0}\right)=1 / 2$.

We turn to the solidity of $f_{1}$. By I.3.7, $f$ is $\theta$-relative with $\theta=\theta_{\varphi}$. By symmetry and by I.3.7, it suffices to show that $f_{1}$ is $\left(\theta_{1}, q\right)$-relative with $\left(\theta_{1}, q\right)$ depending only on $\varphi$. We show that one can choose $q=r_{0} / 3$.

We write $\delta=\delta_{G}, \delta_{1}=\delta_{G_{1}}, \delta^{\prime}=\delta_{G^{\prime}}, \delta_{1}^{\prime}=\delta_{G_{1}^{\prime}}$. Then

$$
\delta(x)=\delta_{1}(x) \wedge|x|, \quad \delta^{\prime}(y)=\delta_{1}^{\prime}(y) \wedge|y| .
$$

Set $q=r_{0} / 3$, and let $a \in G_{1}, b \in B\left(a, q \delta_{1}(a)\right)$. It suffices to find an estimate

$$
\frac{\left|f_{1} a-f_{1} b\right|}{\delta_{1}^{\prime}(f a)} \leq \theta_{1}\left(\frac{|a-b|}{\delta_{1}(a)}\right)
$$

with some $\theta_{1}$ with $\theta_{1}(t) \rightarrow 0$ as $t \rightarrow 0$. By continuity we may assume that $a \neq 0 \neq b$. We consider two cases.

Case 1. $|a| \geq r_{0} / 2$. If $\delta(a)=|a|$,

$$
\delta_{1}(a) \leq 1+|a| \leq\left(2 / r_{0}+1\right)|a|<\delta(a) / q .
$$

If $\delta(a)=\delta_{1}(a)$, this is trivially true. Hence we have $|a-b|<\delta(a)$. Since $f$ is $\theta$-relative, this implies

$$
\frac{|f a-f b|}{\delta_{1}^{\prime}(f a)} \leq \frac{|f a-f b|}{\delta^{\prime}(f a)} \leq \theta\left(\frac{|a-b|}{\delta(a)}\right) \leq \theta\left(\frac{|a-b|}{q \delta_{1}(a)}\right) .
$$

Thus (2.20) holds with $\theta_{1}(t)=\theta(t / q)$. 
Case 2. $|a|<r_{0} / 2$. Now $\delta(a)=|a|$. We consider two subcases.

Subcase 2a. $|a-b| \leq|a|^{2}$. We have

$$
\frac{|f a-f b|}{\delta_{1}^{\prime}(f a)} \leq \frac{|f a-f b|}{\delta^{\prime}(f a)} \leq \theta\left(\frac{|a-b|}{|a|}\right) .
$$

Since $\delta_{1}(a) \leq 1+|a|<2$, we obtain

$$
\frac{|a-b|^{2}}{|a|^{2}} \leq|a-b|<2 \frac{|a-b|}{\delta_{1}(a)} .
$$

Hence (2.20) holds with $\theta_{1}(t)=\theta(\sqrt{2 t})$.

Subcase $2 \mathrm{~b} .|a-b| \geq|a|^{2}$. Since

$$
|a-b| \leq q \delta_{1}(a) \leq q(1+|a|)<r_{0} / 2,
$$

we have $|b|<r_{0}$ and

$$
|b| \leq|a|+|a-b| \leq 2 \sqrt{|a-b|} .
$$

By (2.17) this yields

$$
|f a-f b| \leq|f a|+|f b| \leq 2 \psi(2 \sqrt{|a-b|}) .
$$

Since

$$
\delta_{1}^{\prime}(f a) \geq 1-|f a| \geq 1-\psi(|a|) \geq 1 / 2, \quad \delta_{1}(a) \leq 1+|a| \leq 1+r_{0} / 2 \leq 5 / 4,
$$

we obtain $(2.20)$ with $\theta_{1}(t)=4 \psi(\sqrt{5 t})$. व

2.21. Theorem. Suppose that $x_{0}$ is an isolated boundary point of $G$ and that $f: G \rightarrow G^{\prime}$ is $\varphi$-FQC. Then $f$ has a limit $y_{0} \in \dot{E}^{\prime}$ at $x_{0}$, and $y_{0}$ is an isolated boundary point of $\partial G^{\prime}$. Setting $f_{1}\left(x_{0}\right)=y_{0}$ we obtain an extension of $f$ to a homeomorphism $f_{1}: G \cup\left\{x_{0}\right\} \rightarrow G^{\prime} \cup\left\{y_{0}\right\}$. If $x_{0} \neq \infty$ and $y_{0} \neq \infty$, then $f_{1}$ is $\varphi_{1}-\mathrm{FQC}$ with $\varphi_{1}=\varphi_{1}(\varphi)$.

Proof. This is an easy corollary of 2.7. 口

2.22. Remark. In the QC theory of $R^{n}$ it is customary to allow the possibilities $\infty \in G$ and $\infty \in G^{\prime}$. In the free theory this would involve technical complications, since we have not defined the QH metric of such domains. One can usually reduce the situation to the case $G \subset E, G^{\prime} \subset E^{\prime}$ by auxiliary inversions. 


\section{Neargeodesics}

3.1. Terminology. Let $G \neq E$ and $c \geq 1$. An $\operatorname{arc} \gamma \subset G$ is a $c$-neargeodesic in $G$, if $\gamma$ is $c$-quasiconvex in the QH metric $k=k_{G}$. In other words, the inequality

$$
l_{k}(\gamma[x, y]) \leq c k(x, y)
$$

holds for each pair $x, y \in \gamma$. Thus $\gamma$ is a QH geodesic if and only if it is a 1-neargeodesic. The arc $\gamma$ may be closed, open or half open.

We showed in I.2.9 that geodesics do not always exist. In this section we shall prove two existence theorems for neargeodesics. In the first result we join two points in $G$, in the second one a point of $G$ to $\partial G$.

3.2. Lemma. Suppose that $a \in G \neq E$, that $0<t \leq 1 / 2$, and that $\gamma$ is a rectifiable arc in $\bar{B}(a, t \delta(a))$. Then

$$
\frac{1}{1+t} \leq \frac{l_{k}(\gamma) \delta(a)}{l(\gamma)} \leq \frac{1}{1-t}
$$

Moreover, every line segment in $\bar{B}(a, t \delta(a))$ is a $c$-neargeodesic in $G$ with $c=$ $(1+2 t)^{2}$.

Proof. Since

$$
\delta(a)(1-t) \leq \delta(x) \leq \delta(a)(1+t)
$$

for all $x \in \bar{B}(a, t \delta(a))$, the inequalities follow directly by integration. Suppose that $\gamma=[x, y] \subset \bar{B}(a, t \delta(a))$. Then the second inequality and I.2.2(3) yield

$$
l_{k}(\gamma) \leq(1+2 t)|x-y| / \delta(a) \leq(1+2 t)^{2} k(x, y) \text {. }
$$

3.3. Theorem. Let $a, b \in G \neq E$ and let $c>1$. Then there is a $c$ neargeodesic joining $a$ and $b$ in $G$.

Proof. For $q>0$ we write $c_{1}=c_{1}(q)=(1+2 q)^{2}$. Choose $q_{0}>0$ such that

$$
q_{0} \leq k(a, b) / 10, \quad c_{1}\left(q_{0}\right)<5 / 4 .
$$

Then $q_{0}<(\sqrt{5}-2) / 4<1 / 8$. We shall prove the theorem by constructing for every $q \leq q_{0}$ an $\operatorname{arc} \beta$ joining $a$ and $b$ such that $\beta$ is a $c(q)$-neargeodesic, where $c(q) \rightarrow 1$ as $q \rightarrow 0$.

Let $0<q \leq q_{0}$. Choose an arc $\gamma$ joining $a$ and $b$ in $G$ such that $l_{k}(\gamma) \leq$ $k(a, b)+q^{2}$. Then

$$
l_{k}(\gamma[x, y]) \leq k(x, y)+q^{2}
$$

for all $x, y \in \gamma$, since assuming $x \in \gamma[a, y]$ we have

$$
k(a, x)+l_{k}(\gamma[x, y])+k(y, b) \leq l_{k}(\gamma) \leq k(a, x)+k(x, y)+k(y, b)+q^{2} .
$$

Since $q \leq k(a, b) / 10$, we can choose a number $\lambda=\lambda(q)$ such that 
(1) $q / 4 \leq \lambda \leq q / 2$,

(2) $l_{k}(\gamma)=n \lambda$

for some positive integer $n$. Divide $\gamma$ by successive points $a=x_{0}, \ldots, x_{n}=b$ to subarcs $\gamma_{j}=\gamma\left[x_{j-1}, x_{j}\right]$ with $l_{k}\left(\gamma_{j}\right)=\lambda$. Setting

$$
\beta_{j}=\left[x_{j-1}, x_{j}\right], \quad \beta=\cup\left\{\beta_{j}: 1 \leq j \leq n\right\}
$$

we show that $\beta$ is the desired arc.

Let $x, y \in \beta, x \neq y$. Although we have not yet shown that $\beta$ is an arc, the $\mathrm{QH}$ length $l_{k}(\beta[x, y])$ is defined in the obvious way, as soon as we fix $i$ and $j$ with $x \in \beta_{i}, y \in \beta_{j}$. Setting

$$
p(x, y)=\frac{l_{k}(\beta[x, y])}{k(x, y)}
$$

we must find an upper bound $p(x, y) \leq c(q)$ with $c(q) \rightarrow 1$ as $q \rightarrow 0$. We consider four cases.

Case 1. For some $i, \beta_{i}$ contains $x$ and $y$. Since $k\left(x_{i-1}, x_{i}\right) \leq \lambda \leq q / 2<1$, I.2.5 gives $\left|x_{i-1}-x_{i}\right| \leq q \delta\left(x_{i}\right)$. Hence 3.2 implies that $p(x, y) \leq(1+2 q)^{2}=c_{1}(q)$.

Case 2. $x$ and $y$ are vertices of $\beta$, say $x=x_{i}, y=x_{i+s}, s \geq 1$. Using Case 1 and (3.5) we obtain

$$
\begin{aligned}
l_{k}(\beta[x, y]) & =\sum_{j=1}^{s} l_{k}\left(\beta_{i+j}\right) \leq c_{1} \sum_{j=1}^{s} k\left(x_{i+j-1}, x_{i+j}\right) \leq c_{1} \sum_{j=1}^{s} l_{k}\left(\gamma_{i+j}\right) \\
& =c_{1} l_{k}(\gamma[x, y]) \leq c_{1} k(x, y)+c_{1} q^{2} .
\end{aligned}
$$

On the other hand, since $q<1 / 8,(3.5)$ implies

$$
k(x, y) \geq l_{k}(\gamma[x, y])-q^{2}=s \lambda-q^{2} \geq q / 4-q^{2}>q / 8 .
$$

Hence $p(x, y) \leq c_{1}+8 c_{1} q=c_{2}(q)$.

Case 3. There are $i \geq 1$ and $s \geq i+2$ such that $x \in \beta_{i}, y \in \beta_{s}$. Using Case 2 we obtain

$$
\begin{aligned}
l_{k}(\beta[x, y]) & =l_{k}\left(\beta\left[x_{i-1}, x_{s}\right]\right)-l_{k}\left(\beta\left[x_{i-1}, x\right]\right)-l_{k}\left(\beta\left[y, x_{s}\right]\right) \\
& \leq c_{2} k\left(x_{i-1}, x_{s}\right)-k\left(x_{i-1}, x\right)-k\left(y, x_{s}\right) \\
& \leq c_{2} k(x, y)+\left(c_{2}-1\right)\left[k\left(x_{i-1}, x\right)+k\left(y, x_{s}\right)\right] .
\end{aligned}
$$

Here

$$
k\left(x_{i-1}, x\right) \leq l_{k}\left(\beta_{i}\right) \leq c_{1} k\left(x_{i-1}, x_{i}\right) \leq c_{1} \lambda \leq c_{1} q / 2,
$$


and a similar estimate is valid for $k\left(y, x_{s}\right)$. These and (3.5) yield

$$
\begin{aligned}
k(x, y) & \geq k\left(x_{i-1}, x_{s}\right)-k\left(x_{i-1}, x\right)-k\left(y, x_{s}\right) \\
& \geq l_{k}\left(\gamma\left[x_{i-1}, x_{s}\right]\right)-q^{2}-2 c_{1} \lambda \geq\left(3-2 c_{1}\right) \lambda-q^{2} \geq\left(5-4 c_{1}\right) q / 8
\end{aligned}
$$

where we also made use of the inequalities $\lambda \geq q / 4$ and $q \leq 1 / 8$. By (3.4), the right-hand side is positive. Consequently,

$$
p(x, y) \leq c_{2}+\frac{8 c_{1}\left(c_{2}-1\right)}{5-4 c_{1}}=c_{3}(q) \rightarrow 1
$$

as $q \rightarrow 0$. We have also proved that $\gamma$ is an arc.

Case 4. There is $i$ such that $x \in \beta_{i}$ and $y \in \beta_{i+1}$. If $x=x_{i-1}$ or if $y=x_{i+1}$, we are in Case 3. The general situation is reduced to this special case as follows:

We may assume that $x \neq x_{i} \neq y$. For $K \geq 1$ let $g: E \rightarrow E$ be the similarity defined by $g u=x_{i}+K\left(u-x_{i}\right)$. We can choose $K$ such that $g x \in \beta_{i}, g y \in \beta_{i+1}$, and either $g x=x_{i-1}$ or $g y=x_{i+1}$. In Case 1 we showed that $\beta_{i} \cup \beta_{i+1} \subset$ $\bar{B}\left(x_{i}, q \delta\left(x_{i}\right)\right)$. Applying 3.2 twice we obtain

$$
l_{k}(\beta[x, y]) \leq \frac{l(\beta[x, y])}{(1-q) \delta\left(x_{i}\right)}=\frac{l(\beta[g x, g y])}{K(1-q) \delta\left(x_{i}\right)} \leq \frac{(1+q) l_{k}(\beta[g x, g y])}{K(1-q)}
$$

By Case 3 we have $l_{k}(\beta[g x, g y]) \leq c_{3} k(g x, g y)$. These estimates and I.2.2 yield

$$
\begin{aligned}
l_{k}(\beta[x, y]) & \leq \frac{(1+q) c_{3}|g x-g y|}{K(1-q)^{2} \delta\left(x_{i}\right)}=\frac{(1+q) c_{3}|x-y|}{(1-q)^{2} \delta\left(x_{i}\right)} \leq \frac{(1+q)(1+2 q) c_{3} k(x, y)}{(1-q)^{2}} \\
& =c_{4}(q) k(x, y)
\end{aligned}
$$

where $c_{4}(q) \rightarrow 1$ as $q \rightarrow 0$. ㅁ

3.6. Terminology. A half open arc $\gamma$ in a domain $G$ is an endcut of $G$ if $\bar{\gamma}$ is a closed arc with one endpoint in $\partial G$.

We want to show that each point $x_{0}$ in $G \neq E$ can be joined to $\partial G$ by a neargeodesic endcut. If $\operatorname{dim} E<\infty$ this is easy: We choose $y_{0} \in \partial G$ with $\left|y_{0}-x_{0}\right|=\delta\left(x_{0}\right)$. Then $\left[x_{0}, y_{0}\right)$ is a 3 -neargeodesic by Lemma 3.9 below. In the general case there is no nearest point $y_{0}$, and we must replace $\left[x_{0}, y_{0}\right)$ by a broken line consisting of a countable number of line segments.

We first prove some elementary inequalities in Banach spaces.

3.7. Lemma. Suppose that $a, b \in G \neq E$ with $|a-b| \leq \delta(a)$ and that $x \in[a, b]$. Then $|x-b| \leq \delta(x)$ and $\delta(b) \leq 2 \delta(x)$. 
Proof. Elementary estimates give

$$
\begin{gathered}
|x-a|+|x-b|=|a-b| \leq \delta(a) \leq \delta(x)+|x-a|, \\
\delta(b) \leq \delta(x)+|x-b| \leq 2 \delta(x) .
\end{gathered}
$$

3.8. Lemma. Let $x_{0}, x_{1}, x_{2} \in E$ with $2\left|x_{1}-x_{2}\right| \leq\left|x_{0}-x_{1}\right| \leq\left|x_{0}-x_{2}\right|$, and let $x \in\left[x_{0}, x_{1}\right], y \in\left[x_{1}, x_{2}\right]$. Then

$$
\left|x-x_{1}\right| \leq 2|x-y|, \quad\left|x_{1}-y\right| \leq 2|x-y| .
$$

Proof. We normalize $x_{0}=0,\left|x_{1}\right|=1$. Then $2\left|x_{1}-x_{2}\right| \leq 1 \leq\left|x_{2}\right|$. Using a similarity of the form $f(x)=x_{1}+K\left(x-x_{1}\right)$ we see that it suffices to consider the cases where either $x=0$ or $y=x_{2}$.

Suppose first that $x=0$. Then

$$
|x-y|=|y| \geq\left|x_{1}\right|-\left|x_{1}-y\right| \geq 1-\left|x_{1}-x_{2}\right| \geq 1 / 2
$$

and hence

$$
\left|x-x_{1}\right|=1 \leq 2|x-y|, \quad\left|x_{1}-y\right| \leq\left|x_{1}-x_{2}\right| \leq 1 / 2 \leq|x-y| .
$$

Next assume that $y=x_{2}$. Now

$$
|x-y| \geq|y|-|x| \geq 1-|x|=\left|x-x_{1}\right|
$$

which implies the first inequality. If $\left|x_{1}-y\right| \geq 2\left|x_{1}-x\right|$, then

$$
\left|x_{1}-y\right| \leq\left|x_{1}-x\right|+|x-y| \leq\left|x_{1}-y\right| / 2+|x-y| .
$$

If $\left|x_{1}-y\right| \leq 2\left|x_{1}-x\right|$, then

$$
\left|x_{1}-y\right| / 2 \leq\left|x_{1}-x\right|=1-|x| \leq|y|-|x| \leq|y-x| .
$$

In both cases we obtain the second inequality. $\square$

3.9. Lemma. Suppose that $a, b \in G \neq E$ and that $|a-b| \leq \delta(a)$. Then

$$
l_{k}([a, b]) \leq 3 \ln \left(1+\frac{|a-b|}{\delta(b)}\right),
$$

and $[a, b]$ is a 3 -neargeodesic. 
Proof. . For each $x \in[a, b]$ and $y \in \partial G$ we have

$|b-x|=|a-b|-|a-x| \leq \delta(a)-|a-x| \leq \delta(x), \quad \delta(b) \leq \delta(x)+|b-x| \leq 2 \delta(x)$,

and hence

$$
|x-b|+\delta(b) \leq 3 \delta(x)
$$

Consequently,

$$
l_{k}([a, b]) \leq 3 \int_{0}^{|a-b|} \frac{d t}{\delta(b)+t}=3 \ln \left(1+\frac{|a-b|}{\delta(b)}\right) .
$$

The last statement follows now from I.2.2(1). $\square$

3.10. Theorem. Suppose that $x_{0} \in G \neq E$ and that $\varepsilon>0$. Then there is an endcut $\gamma$ of $G$ from $x_{0}$ such that

(1) $\gamma$ is a $c_{0}$-neargeodesic with a universal $c_{0}$,

(2) $\gamma \subset B\left(x_{0},(1+\varepsilon) \delta\left(x_{0}\right)\right)$.

Proof. We may assume that $\varepsilon \leq 1 / 4$. For positive integers $j$ set $\varepsilon_{j}=2^{-j} \varepsilon$. We construct inductively a sequence of points $x_{0}, x_{1}, \ldots$ in $\bar{G}$ as follows: Suppose that $x_{0}, \ldots, x_{i}$ have been chosen. If $x_{i} \in \partial G$, the process stops. If $x_{i} \in G$, we choose a point $y_{i+1} \in \partial G$ with $\left|y_{i+1}-x_{i}\right|<\left(1+\varepsilon_{i+1}\right) \delta\left(x_{i}\right)$, and let $x_{i+1}$ be the unique point in $\left[x_{i}, y_{i+1}\right] \cap S\left(x_{i}, \delta\left(x_{i}\right)\right)$. Then

$$
\delta\left(x_{i+1}\right)<\varepsilon_{i+1} \delta\left(x_{i}\right)
$$

Writing $\gamma_{i}=\left[x_{i-1}, x_{i}\right)$ we claim that the union $\gamma$ of all $\gamma_{i}$ is the desired endcut. As in the proof of 3.3, we can in the obvious way define the QH length of $\gamma[x, y]$ for $x \in \gamma_{i}, y \in \gamma_{j}$ as soon as $i$ and $j$ are fixed. We shall show that

$$
l_{k}(\gamma[x, y]) \leq c_{0} k(x, y)
$$

for all $x, y \in \gamma$ with a universal constant $c_{0}$. This will imply that the $\operatorname{arcs} \gamma_{j}$ are disjoint. Moreover, since

$$
\sum_{j} l\left(\gamma_{j}\right)=\sum_{j} \delta\left(x_{j-1}\right)<\delta\left(x_{0}\right)\left(1+\sum_{j=1}^{\infty} \varepsilon_{j}\right)=\delta\left(x_{0}\right)(1+\varepsilon),
$$

$\gamma$ lies in $B\left(x_{0},(1+\varepsilon) \delta\left(x_{0}\right)\right)$, and $\gamma$ is rectifiable. Since $E$ is complete, this implies that $\gamma$ is an endcut of $G$. Thus it suffices to verify (3.12). We consider three cases.

Case 1. For some $i, \gamma_{i}$ contains $x$ and $y$. Now (3.12) follows from 3.9. 
Case 2. For some $i, x_{i} \in \gamma_{i}$ and $y \in \gamma_{i+1}$. Now 3.9 gives

$$
l_{k}(\gamma[x, y]) \leq 3 \ln \left(1+\frac{\left|x-x_{i}\right|}{\delta\left(x_{i}\right)}\right)+3 \ln \left(1+\frac{\left|x_{i}-y\right|}{\delta(y)}\right) .
$$

Applying 3.8 with the substitution

$$
\left(x_{0}, x_{1}, x_{2}, x, y\right) \mapsto\left(x_{i-1}, x_{i}, y_{i+1}, x, y\right)
$$

we obtain

$$
\left|x-x_{i}\right| \vee\left|x_{i}-y\right| \leq 2|x-y|
$$

Since

$$
\delta(y) \leq\left|y-y_{i+1}\right|<\left(1+\varepsilon_{i+1}\right) \delta\left(x_{i}\right) \leq 9 \delta\left(x_{i}\right) / 8,
$$

and since $\ln (1+t a) \leq t \ln (1+a)$ for $t \geq 1$ and $a \geq 0$, these inequalities and I.2.2 yield the estimate

$$
l_{k}(\gamma[x, y]) \leq\left(\frac{27}{4}+6\right) \ln \left(1+\frac{|x-y|}{\delta(y)}\right) \leq 13 k(x, y) .
$$

Case 3. For some $i \geq 1$ and $s \geq 2, x \in \gamma_{i}$ and $y \in \gamma_{i+s}$. By 3.9 we have $l_{k}(\gamma[x, y]) / 3 \leq \ln \left(1+\frac{\left|x_{i}-x\right|}{\delta\left(x_{i}\right)}\right)+\sum_{j=1}^{s-1} \ln \left(1+\frac{\delta\left(x_{i+j-1}\right)}{\delta\left(x_{i+j}\right)}\right)+\ln \left(1+\frac{\left|x_{i+s-1}-y\right|}{\delta(y)}\right)$. By (3.11) we obtain

Writing

$$
\ln \left(1+\frac{\delta\left(x_{i+j-1}\right)}{\delta\left(x_{i+j}\right)}\right) \leq \ln \frac{\delta\left(x_{i+j-1}\right)}{\delta\left(x_{i+j}\right)}+\ln \left(1+\varepsilon_{i+j}\right) \leq \ln \frac{\delta\left(x_{i+j-1}\right)}{\delta\left(x_{i+j}\right)}+\varepsilon_{i+j} .
$$

these inequalities yield

$$
\alpha=\ln \frac{\delta\left(x_{i}\right)+\left|x_{i}-x\right|}{\delta(x)}, \quad \beta=\ln \frac{\delta(y)+\left|y-x_{i+s-1}\right|}{\delta\left(x_{i+s-1}\right)},
$$

$$
l_{k}(\gamma[x, y]) / 3 \leq \alpha+\beta+\varepsilon+\ln \frac{\delta(x)}{\delta(y)} .
$$

Furthermore, Lemma 3.7 implies that

$$
\delta\left(x_{i}\right)+\left|x_{i}-x\right| \leq 2 \delta(x)+\delta(x)=3 \delta(x),
$$

and hence $\alpha \leq \ln 3$. Since $\left|y-x_{i+s-1}\right| \leq \delta\left(x_{i+s-1}\right)$, we have

$$
\delta(y) \leq \delta\left(x_{i+s-1}\right)+\left|y-x_{i+s-1}\right| \leq 2 \delta\left(x_{i+s-1}\right),
$$

and thus $\beta \leq \ln 3$. Since $\varepsilon<1$, these estimates imply

$$
l_{k}(\gamma[x, y]) \leq M+3 \ln \frac{\delta(x)}{\delta(y)},
$$

where $M=3(1+2 \ln 3)$. We also see that

$$
\delta(y) \leq 2 \delta\left(x_{i+s-1}\right) \leq 2 \varepsilon_{1} \delta\left(x_{i}\right)=\varepsilon \delta\left(x_{i}\right) \leq \delta\left(x_{i}\right) / 4 \leq \delta(x) / 2 .
$$

Hence (3.13) and I.2.2 give (3.12) with $c_{0}=M / \ln 2+3<17$. $\square$ 


\section{Coarse length and CQH maps}

4.1. Introduction to Section 4. We first introduce the concept of the coarse length of an arc, which will be our main tool when studying the boundary properties of FQC and more general maps. We also consider coarsely bilipschitz maps, which in the QH case will be called coarsely quasihyperbolic or $\mathrm{CQH}$. The $\mathrm{CQH}$ maps include the solid maps, and hence the FQC maps.

The general idea in the coarse theory is that we forget what happens with small distances. Related concepts have been considered by M. Gromov [Gr, p. 186] and several others.

4.2. Terminology. Let $\gamma$ be an arc in a metric space $X$. The arc may be closed, open or half open. Let $\bar{x}=\left(x_{0}, \ldots, x_{n}\right), n \geq 1$, be a finite sequence of successive points of $\gamma$. For $h \geq 0$ we say that $\bar{x}$ is $h$-coarse if $\left|x_{j-1}-x_{j}\right| \geq h$ for all $1 \leq j \leq n$. Let $\Phi(\gamma, h)$ be the family of all $h$-coarse sequences of $\gamma$. Set

$$
\begin{aligned}
s(\bar{x}) & =\sum_{j=1}^{n}\left|x_{j-1}-x_{j}\right|, \\
l(\gamma, h) & =\sup \{s(\bar{x}): \bar{x} \in \Phi(\gamma, h)\}
\end{aligned}
$$

with the agreement that $l(\gamma, h)=0$ if $\Phi(\gamma, h)=\emptyset$. The number $l(\gamma, h)$ is the $h$-coarse length of $\gamma$.

In this paper we shall use this concept in the case where $X$ is a domain $G \neq E$ equipped with the $\mathrm{QH}$ metric $k$. We let $l_{k}(\gamma, h)$ denote the $h$-coarse $\mathrm{QH}$ length of $\gamma$.

This concept is useful in the theory of solid and FQC maps $f: G \rightarrow G^{\prime}$, because we can compare suitable coarse lengths of an arc $\gamma \subset G$ and its image $f \gamma$. The ordinary length is useless, since $f$ need not preserve the rectifiability of an arc.

We list some elementary properties of the coarse length:

4.3. Lemma. Let $\gamma$ be an arc in a metric space, and let $h \geq 0$.

(1) If $\gamma$ is a closed arc and $h>0$, then $l(\gamma, h)<\infty$.

(2) $l(\gamma, h)$ is decreasing in $h$.

(3) $l(\gamma, 0)=l(\gamma)$ is the ordinary length of $\gamma$.

(4) $l(\gamma, h)=0$ for $h>d(\gamma)$.

(5) $d(\gamma) \leq h \vee l(\gamma, h)$.

(6) $\gamma^{\prime} \subset \gamma$ implies $l\left(\gamma^{\prime}, h\right) \leq l(\gamma, h)$.

(7) $l(\gamma, h)$ is the supremum of $s(\bar{x})$ over all $\bar{x}=\left(x_{0}, \ldots, x_{n}\right)$ which satisfy the condition $h \leq\left|x_{j-1}-x_{j}\right|<2 h$ for all $1 \leq j \leq n$.

Proof. The property (1) follows easily from the compactness of $\gamma$, and the properties from (2) to (6) are direct consequences of the definition. Each $h$-coarse $\bar{x}$ has obviously a refinement $\bar{y}$ such that $h \leq\left|y_{j-1}-y_{j}\right|<2 h$ for all $j$. This implies (7). ם 
4.4. Remark. The coarse length is not additive. If $\gamma$ is divided to two subarcs $\gamma_{1}, \gamma_{2}$, we usually have $l(\gamma, h) \neq l\left(\gamma_{1}, h\right)+l\left(\gamma_{2}, h\right)$. One can easily prove the inequalities

$$
l\left(\gamma_{1}, 2 h\right)+l\left(\gamma_{2}, 2 h\right)-h \leq l(\gamma, h) \leq l\left(\gamma_{1}, h\right)+l\left(\gamma_{2}, h\right)+2 h,
$$

but they are not needed in this paper.

The following result will be needed in Section 6:

4.5. Lemma. Let $G \neq E$ and let $\gamma$ be an arc in $G \cap(\partial G+\bar{B}(r))$. If $0 \leq h \leq R$ and $l_{k}(\gamma, h) \leq R$, then $d(\gamma) \leq M R r$, where $M=M(h)$ is increasing in $h$. If $h=0$, we have $l(\gamma) \leq R r$.

Proof. The case $h=0$ is easy, since

$$
\frac{l(\gamma)}{r} \leq \int_{\gamma} \frac{|d x|}{\delta(x)}=l_{k}(\gamma) \leq R
$$

Assume that $h>0$. We may assume that $\gamma$ is a closed arc with endpoints $a_{0}, a_{1}$. Replacing $\gamma$ by a subarc we may assume that $d(\gamma)=\left|a_{0}-a_{1}\right|$. We show that the lemma is true with $M=2\left(e^{h}-1\right) / h$, which is easily seen to be increasing in $h$.

If $\gamma \subset B_{k}\left(a_{0}, h\right)$, then I.2.2 implies that

$$
d(\gamma)=\left|a_{0}-a_{1}\right| \leq \delta\left(a_{0}\right)\left(e^{k\left(a_{0}, a_{1}\right)}-1\right) \leq r\left(e^{h}-1\right) \leq M r h \leq M r R .
$$

Suppose that $\gamma \not \subset B_{k}\left(a_{0}, h\right)$. Choose a sequence $\bar{x}=\left(x_{0}, \ldots, x_{n}\right)$ of successive points of $\gamma$ such that $x_{0}=a_{0}, k\left(x_{j-1}, x_{j}\right)=h$ for $1 \leq j \leq n$, and $k\left(x_{n}, a_{1}\right)<h$. Then $n \geq 1$ and

$$
n h=s(\bar{x}) \leq l_{k}(\gamma, h) \leq R .
$$

By I.2.2 this implies

$$
d(\gamma)=\left|a_{0}-a_{1}\right| \leq s(\bar{x})+\left|x_{n}-a_{1}\right| \leq(n+1) r\left(e^{h}-1\right) \leq M R r . \text { 口 }
$$

4.6. Terminology. Let $M \geq 0$ and $C \geq 0$. A map $f: X \rightarrow Y$ is $C$-coarsely $M$-Lipschitz if

$$
|f x-f y| \leq M|x-y|+C
$$

for all $x, y \in X$. If $f$ is an embedding and if $f$ and $f^{-1}: f X \rightarrow X$ are $C$-coarsely $M$-Lipschitz with $M \geq 1$, we say that $f$ is $C$-coarsely $M$-bilipschitz, abbreviated $(M, C)$-CBL. This means that

$$
(|x-y|-C) / M \leq|f x-f y| \leq M|x-y|+C
$$

for all $x, y \in X$. In $\left[\mathrm{V}_{3}\right]$ the CBL maps were called roughly bilipschitz.

One could also consider maps satisfying (4.7) which are not injective or continuous. However, it is often helpful to be able to consider the inverse map $f^{-1}$. It seems to the author that one could develop an analogous theory for one-to-many "maps" (relations), but this would involve technical complications.

Recall from I.2.8 that a metric space $X$ is $c$-quasiconvex if each pair of points $x, y \in X$ can be joined by an arc $\gamma \subset X$ with $l(\gamma) \leq c|x-y|$. 
4.8. Theorem. Suppose that $X$ is $c$-quasiconvex, that $d(X)=\infty$ and that $f: X \rightarrow Y$ is a map. Then the following conditions are quantitatively equivalent: (1) $f$ is $C$-coarsely $M$-Lipschitz.

(2) There are $t_{1} \geq 0$ and $M_{1} \geq 0$ such that $|f x-f y| \leq M_{1}|x-y|$ whenever $x, y \in X$ and $|x-y| \geq t_{1}$.

(3) There are $t_{0}>0$ and $M_{0} \geq 0$ such that $|f x-f y| \leq M_{0}$ whenever $x, y \in X$ and $|x-y| \leq t_{0}$.

Proof. We show that (1) $\Rightarrow(2) \Rightarrow(3) \Rightarrow(1)$.

If (1) holds and if $|x-y| \geq C$, then

$$
|f x-f y| \leq M|x-y|+C \leq(M+1)|x-y| \text {. }
$$

Hence (2) is true with $t_{1}=C, M_{1}=M+1$.

We next show that (2) implies (3) with $t_{0}=t_{1}+1$ and $M_{0}=5 M_{1}\left(t_{1}+1\right)$. Assume that $x, y \in X$ with $|x-y| \leq t_{0}$. Since $X$ is connected and since $d(X)=$ $\infty$, there is a point $z$ in $X$ such that $|z-x|=2 t_{0}$. Then $t_{0} \leq|z-y| \leq 3 t_{0}$, and we obtain

$$
|f x-f y| \leq|f x-f z|+|f z-f y| \leq M_{1}|x-z|+M_{1}|z-y| \leq 5 M_{1}\left(t_{1}+1\right) .
$$

Finally assume that (3) is true. Let $x, y \in X$. Choose an arc $\gamma$ joining $x$ and $y$ with $l(\gamma) \leq c|x-y|$. Let $k \geq 0$ be the unique integer with $k t_{0}<l(\gamma) \leq(k+1) t_{0}$. Choose successive points $x=x_{0}, \ldots, x_{k+1}=y$ such that each subarc $\gamma\left[x_{j-1}, x_{j}\right]$ has length at most $t_{0}$. Then $\left|x_{j-1}-x_{j}\right| \leq t_{0}$ and hence $\left|f x_{j-1}-f x_{j}\right| \leq M_{0}$. This implies

$$
|f x-f y| \leq(k+1) M_{0} \leq M_{0} l(\gamma) / t_{0}+M_{0} \leq c M_{0}|x-y| / t_{0}+M_{0},
$$

which gives (1) with $M=c M_{0} / t_{0}, C=M_{0}$.

4.9. Theorem. Suppose that $f: X \rightarrow Y$ is a $C$-coarsely $M$-Lipschitz embedding, that $\gamma$ is an arc in $X$ and that $h \geq 0$. Then for $h_{1}=M(h \vee C)+C$ we have

$$
l\left(f \gamma, h_{1}\right) \leq(M+1) l(\gamma, h) .
$$

Proof. Let $\bar{y}=\left(y_{0}, \ldots, y_{n}\right)$ be a $h_{1}$-coarse sequence of $f \gamma$. Writing $x_{j}=$ $f^{-1}\left(y_{j}\right)$ we obtain a sequence $\bar{x}=\left(x_{0}, \ldots, x_{n}\right)$. Since

$$
\left|x_{j-1}-x_{j}\right| \geq\left(\left|y_{j-1}-y_{j}\right|-C\right) / M \geq\left(h_{1}-C\right) / M=h \vee C,
$$

$\bar{x}$ is $h$-coarse. Moreover,

$$
s(\bar{y}) \leq \sum_{j=1}^{n}\left(M\left|x_{j-1}-x_{j}\right|+C\right),
$$

where $\left|x_{j-1}-x_{j}\right| \geq h \vee C \geq C$, and hence

$$
s(\bar{y}) \leq(M+1) s(\bar{x}) \leq(M+1) l(\gamma, h),
$$

which implies the theorem. $\square$ 
4.10. Terminology. Let $h \geq 0$ and $c \geq 1$. A metric space $X$ is $h$-coarsely $c$-quasiconvex if each pair of points $x, y \in X$ can be joined by an arc $\gamma$ with

$$
l(\gamma, h) \leq c|x-y|
$$

In particular, an arc $\gamma$ is $h$-coarsely $c$-quasiconvex if

$$
l(\gamma[x, y], h) \leq c|x-y|
$$

for all $x, y \in \gamma$. The case where $\gamma$ is an arc in a domain $G \neq E$ with the $\mathrm{QH}$ metric plays an important role in the rest of the paper. We say briefly that an arc $\gamma \subset G$ is $(c, h)$-solid in $G$ if it is $h$-coarsely $c$-quasiconvex in the QH metric of $G$. For $h=0$ this means that $\gamma$ is a $c$-neargeodesic.

4.11. Theorem. Suppose that $f: X \rightarrow Y$ is $(M, C)$-CBL and that the arc $\gamma \subset X$ is $h$-coarsely $c$-quasiconvex. Then $f \gamma$ is $h_{1}$-coarsely $c_{1}$-quasiconvex with

$$
h_{1}=M(h \vee 2 c C)+C, \quad c_{1}=2 c M(M+1) .
$$

Proof. Replacing $\gamma$ by a subarc we see that it suffices to show that

$$
l\left(f \gamma, h_{1}\right) \leq c_{1}|f x-f y|
$$

where $x$ and $y$ are the endpoints of $\gamma$. If $|x-y| \geq 2 C$, we have

$$
|f x-f y| \geq(|x-y|-C) / M \geq|x-y| / 2 M .
$$

On the other hand, $l(\gamma, h) \leq c|x-y|$ implies by 4.9 that

$$
l\left(f \gamma, h_{1}\right) \leq(M+1) c|x-y|
$$

and (4.12) follows.

Next assume that $|x-y|<2 C$. It suffices to show that $|f u-f v|<h_{1}$ for all $u, v \in \gamma$, since then $l\left(f \gamma, h_{1}\right)=0$, and (4.12) is trivially true. If $|u-v|<h$, then

$$
|f u-f v|<M h+C \leq h_{1} .
$$

If $|u-v| \geq h$, then

$$
|u-v| \leq l(\gamma, h) \leq c|x-y|<2 c C,
$$

which implies

$$
|f u-f v|<2 c C M+C \leq h_{1} \text {. } \text { 口 }
$$


4.13. Terminology. Recall from I.3.4 that a homeomorphism $f: G \rightarrow G^{\prime}$ between domains $G \neq E$ and $G^{\prime} \neq E^{\prime}$ is $M$-quasihyperbolic or $M$-QH if $f$ is $M$-bilipschitz in the QH metric. Similarly, we say that $f: G \rightarrow G^{\prime}$ is $C$-coarsely $M$-quasihyperbolic, abbreviated $(M, C)$ - $\mathrm{CQH}$, if it is $(M, C)-\mathrm{CBL}$ in the $\mathrm{QH}$ metric. This means that $f$ is a homeomorphism such that

$$
(k(x, y)-C) / M \leq k^{\prime}(f x, f y) \leq M k(x, y)+C
$$

for all $x, y \in G$.

We next give the relation between the $\mathrm{CQH}$ maps and some other classes considered in this paper. In 7.9 and in 7.22 we shall prove the close connection between the CQH maps and the maps which are quasimöbius relative to the boundary. More results on CQH maps will be given in [Vä ${ }_{5}$. For example, the properties $\varphi$-FQC and fully $(M, C)-\mathrm{CQH}$ are quantitatively equivalent.

4.14. Theorem. For a homeomorphism $f: G \rightarrow G^{\prime}$ with $G \neq E, G^{\prime} \neq E^{\prime}$, the following implications are quantitatively true:

$$
M-\mathrm{QH} \Rightarrow \varphi \text {-FQC } \Rightarrow \varphi \text {-solid } \Rightarrow(M, C)-\mathrm{CQH} .
$$

In the last implication, one can choose an arbitrary $C>0$ and then $M=$ $C / \varphi^{-1}(C)$.

Proof. If $f$ is $M-\mathrm{QH}$, then $f$ is $\varphi$-FQC with $\varphi(t)=4 M^{2} t$ by I.4.7. The second implication is trivial. Suppose that $f$ is $\varphi$-solid and that $C>0, c>1$. Then $k^{\prime}(f x, f y) \leq C$ whenever $k(x, y) \leq \varphi^{-1}(C)$. Since $G$ is $c$-quasiconvex in the QH metric, the proof of 4.8 shows that $f$ is $C$-coarsely $M$-Lipschitz in the $\mathrm{QH}$ metric with $M=c C / \varphi^{-1}(C)$. The same is true for $f^{-1}$. Since $c>1$ is arbitrary, the theorem follows. $\square$

4.15. Theorem. Suppose that $G \neq E, G^{\prime} \neq E^{\prime}$, and that $f: G \rightarrow G^{\prime}$ is $(M, C)$-CQH. If $\gamma$ is a $(c, h)$-solid arc in $G$, then the arc $f \gamma$ is $\left(c_{1}, h_{1}\right)$-solid in $G^{\prime}$ with $\left(c_{1}, h_{1}\right)$ depending only on $(c, h, M, C)$. In particular, if $f$ is $\varphi$-solid or $\varphi$-FQC, then $\left(c_{1}, h_{1}\right)$ depends only on $(c, h, \varphi)$.

Proof. This follows from 4.11 and from 4.14 . $\square$

\section{Relative quasisymmetry and quasimöbius}

5.1. Introduction to Section 5. In this section we shall relativize the theory of quasisymmetric and quasimöbius maps. This theory will be applied in later sections to study the properties of CQH maps.

5.2. Terminology. By a triple in a space $X$ we mean an ordered sequence $T=(x, a, b)$ of three distinct points in $X$. The ratio of $T$ is the number

$$
\rho(T)=\frac{|a-x|}{|b-x|}
$$


If $f: X \rightarrow Y$ is an injective map, the image of a triple $T=(x, a, b)$ is the triple $f T=(f x, f a, f b)$.

Suppose that $A \subset X$. A triple $T=(x, a, b)$ in $X$ is said to be a triple in the pair $(X, A)$ if $x \in A$ or if $\{a, b\} \subset A$. Equivalently, both $|a-x|$ and $|b-x|$ are distances from a point in $A$.

Let $\eta:[0, \infty) \rightarrow[0, \infty)$ be a homeomorphism. An embedding $f: X \rightarrow Y$ is said to be $\eta$-quasisymmetric relative to $A$, abbreviated $\eta$-QS rel $A$, if the condition

$$
\rho(f T) \leq \eta(\rho(T))
$$

holds for every triple $T$ in $(X, A)$. Thus quasisymmetry rel $X$ is equivalent to ordinary quasisymmetry.

Analogously, a quadruple in $X$ is an ordered sequence $Q=(a, b, c, d)$ of four distinct points in $X$. The cross ratio of $Q$ is the number

$$
\tau(Q)=|a, b, c, d|=\frac{|a-b||c-d|}{|a-c||b-d|} .
$$

Warning: The order of the points $a, b, c, d$ varies in the literature. In particular, the cross ratio above is written as $|a, d, b, c|$ in $\left[\mathrm{V}_{a_{1}}\right]$. The definition is extended in the well known manner to the case where one of the points is $\infty$. For example,

$$
|a, b, c, \infty|=\frac{|a-b|}{|a-c|}=\rho(a, b, c) .
$$

If $X_{0} \subset \dot{X}$ and if $f: X_{0} \rightarrow \dot{Y}$ is an injective map, the image of a quadruple $Q$ in $X_{0}$ is the quadruple $f Q=(f a, f b, f c, f d)$.

Suppose that $A \subset X_{0} \subset \dot{X}$. We say that a quadruple $Q=(a, b, c, d)$ in $X_{0}$ is a quadruple in the pair $\left(X_{0}, A\right)$ if $\{a, d\} \subset A$ or $\{b, c\} \subset A$. Equivalently, all four distances in the definition of $\tau(Q)$ are (at least formally) distances from a point of $A$.

Let $\eta:[0, \infty) \rightarrow[0, \infty)$ be a homeomorphism and let $A \subset X_{0} \subset \dot{X}$. An embedding $f: X_{0} \rightarrow Y$ is said to be $\eta$-quasimöbius relative to $A$, abbreviated $\eta$-QM rel $A$, if the inequality

$$
\tau(f Q) \leq \eta(\tau(Q))
$$

holds for each quadruple in $\left(X_{0}, A\right)$. Thus $\eta$-QM rel $X_{0}$ is equivalent to ordinary quasimöbius.

5.4. Remarks. 1. Since $|a, b, c, d|=|b, a, d, c|$, an embedding $f: X_{0} \rightarrow \dot{Y}$ is $\eta$-QM rel $A$ as soon as (5.3) holds for each quadruple $(a, b, c, d)$ with $\{a, d\} \subset A$.

2. It is possible to extend the relative concepts to some cases where the map is not everywhere injective. Let us say that a map $f: X \rightarrow Y$ is injective rel $A$ if $f \mid A$ is injective and if $f^{-1} f A=A$. For such maps the definitions of QS and QM rel $A$ still make sense. However, since such maps do not always have inverse maps, we have the difficulties mentioned in 4.6. 
5.5. Relative theory. We shall next give a relative version of the basic quasimöbius theory of $\left[\mathrm{Vä}_{1}\right]$. In most proofs it is sufficient to check that the corresponding proof in the absolute case makes only use of triples and quadruples in the given pair $(X, A)$. In such cases the proof is omitted.

5.6. Theorem. If $f: X \rightarrow Y$ is $\eta$-QS rel $A$, then $f$ is $\theta$-QM rel $A$ with $\theta=\theta_{\eta}$

Proof. As the absolute case [V $\left.\ddot{a ̈}_{1}, 3.2\right]$. 口

5.7. Lemma. Suppose that $f: X \rightarrow Y$ is an embedding, that $A \subset X$ and that $\eta:[0, \infty) \rightarrow[0, \infty)$ is an increasing function such that

(1) $\rho(f T) \leq \eta(\rho(T))$ for each triple $T$ in $(X, A)$,

(2) $\rho\left(f^{-1} T^{\prime}\right) \leq \eta\left(\rho\left(T^{\prime}\right)\right)$ for each triple $T^{\prime}$ in $(f X, f A)$.

Then $f$ is $\eta_{1}$-QS rel $A$ with $\eta_{1}$ depending only on $\eta$.

Proof. Observe that we do not require $\eta(0)=0$. Replacing $\eta$ by a larger function we may assume that $\eta$ is a homeomorphism onto $\left[r_{0}, \infty\right), r_{0}>0$. Setting $t_{0}=1 / r_{0}$ we define an increasing homeomorphism $\eta_{0}:\left(0, t_{0}\right) \rightarrow(0, \infty)$ by $\eta_{0}(t)=$ $\eta^{-1}\left(t^{-1}\right)^{-1}$. Suppose that $T=(x, a, b)$ is a triple in $(X, A)$ with $\rho(T)<t_{0}$. Applying (2) to the ratio $T^{\prime}=(f x, f b, f a)$ gives $1 / \rho(T) \leq \eta\left(\rho\left(T^{\prime}\right)\right)$, which implies $\rho(f T) \leq \eta_{0}(\rho(T))$. Together with (1) this proves the lemma. $\square$

5.8. Lemma. Suppose that $A \subset X_{0} \subset \dot{X}$, that $f: X_{0} \rightarrow \dot{Y}$ is an embedding and that $\eta:[0, \infty) \rightarrow[0, \infty)$ is an increasing function such that

(1) $\tau(f Q) \leq \eta(\tau(Q))$ for each quadruple $Q=(a, b, c, d)$ in $X_{0}$ with $\{a, d\} \subset A$. (2) $\tau\left(f^{-1} Q^{\prime}\right) \leq \eta\left(\tau\left(Q^{\prime}\right)\right)$ for each quadruple $Q^{\prime}=\left(a^{\prime}, b^{\prime}, c^{\prime}, d^{\prime}\right)$ in $f X_{0}$ with $\left\{a^{\prime}, d^{\prime}\right\} \subset f A$.

Then $f$ is $\eta_{1}$-QM rel $A$ with $\eta_{1}$ depending only on $\eta$.

Proof. In view of 5.4.1, the proof is an obvious modification of the proof of 5.7. ㅁ

5.9. Theorem. Suppose that $X$ and $Y$ are bounded spaces, that $A \subset X$, and that $f: X \rightarrow Y$ is $\theta$-QM rel $A$. Suppose also that $\lambda>0, z_{1} \in X$ and $z_{2}, z_{3} \in A$ are such that

$$
\left|z_{i}-z_{j}\right| \geq d(X) / \lambda, \quad\left|f z_{i}-f z_{j}\right| \geq d(Y) / \lambda
$$

for $i \neq j$. Then:

(1) There is a homeomorphism $\mu=\mu_{\theta, \lambda}:[0, \infty) \rightarrow[0, \infty)$ such that

$$
\frac{|f x-f y|}{d(Y)} \leq \mu\left(\frac{|x-y|}{d(X)}\right)
$$

for all $x \in A, y \in X$.

(2) $f$ is $\eta$-QS rel $A$ with $\eta=\eta_{\theta, \lambda}$. 
Proof. The part (1) is proved as the absolute case [Vä1, 2.1]. In the part (2), we must replace the proof of the absolute case [ $\left.\mathrm{Va}_{1}, 3.12\right]$ by the following argument:

We may assume that $f$ is a homeomorphism and that $f^{-1}: Y \rightarrow X$ is $\theta$ $\mathrm{QM}$ rel $f A$. We normalize the situation so that $d(X)=d(Y)=\lambda$ replacing the metric $|a-b|$ of $X$ by $\lambda|a-b| / d(X)$ and similarly in $Y$. By (1), there is a homeomorphism $\varphi=\varphi_{\theta, \lambda}:[0, \infty) \rightarrow[0, \infty)$ such that

$$
\varphi^{-1}(|x-y|) \leq|f x-f y| \leq \varphi(|x-y|)
$$

for all $x \in A, y \in X$.

Suppose that $T=(x, a, b)$ is a triple in $(X, A)$. Since $f^{-1}$ is $\theta$-QM rel $f A$, it follows from 5.7 that it suffices to find an estimate

$$
\rho(f T) \leq \eta(\rho(T))
$$

for some increasing $\eta=\eta_{\theta, \lambda}:[0, \infty) \rightarrow[0, \infty)$. Since $\left|z_{2}-z_{3}\right| \geq 1$, we may assume that $\left|a-z_{2}\right| \geq 1 / 2$. We consider three cases.

Case 1. $|a-x| \geq 1 / 4$. Now $|b-x| \geq 1 / 4 \rho(T)$. Since $b \in A$ or $x \in A$, we have $|f b-f x| \geq \varphi^{-1}(1 / 4 \rho(T))$, and hence (5.10) is true with $\eta(t)=\lambda / \varphi^{-1}(1 / 4 t)$.

Case 2. $\left|b-z_{2}\right| \geq 1 / 8$. Now $\left|f b-f z_{2}\right| \geq \varphi^{-1}(1 / 8)$. The quadruple $Q=$ $\left(x, a, b, z_{2}\right)$ is in $(X, A)$, and $\tau(Q) \leq 2 \lambda \rho(T)$. Since $\tau(f Q) \geq \varphi^{-1}(1 / 8) \rho(f T) / \lambda$, we obtain (5.10) with

$$
\eta(t)=\frac{\lambda \theta(2 \lambda t)}{\varphi^{-1}(1 / 8)}
$$

Case 3. $|a-x| \leq 1 / 4$ and $\left|b-z_{2}\right| \leq 1 / 8$. Now

$$
|b-x| \geq\left|a-z_{2}\right|-|a-x|-\left|z_{2}-b\right| \geq 1 / 8
$$

Hence $|f b-f x| \geq \varphi^{-1}(1 / 8)$, which implies (5.10) with the constant function $\eta(t)=\lambda / \varphi^{-1}(1 / 8)$.

5.11. Theorem. Suppose that $G$ and $G^{\prime}$ are bounded domains and that $c \geq 1$. Suppose also that $x_{0} \in G$ and $x_{0}^{\prime} \in G^{\prime}$ are points with

$$
d(G) \leq c \delta\left(x_{0}\right), \quad d\left(G^{\prime}\right) \leq c \delta^{\prime}\left(x_{0}^{\prime}\right) .
$$

Let $f: \bar{G} \rightarrow \bar{G}^{\prime}$ be a homeomorphism such that $f x_{0}=x_{0}^{\prime}$ and $f G=G^{\prime}$. If $f$ is $\theta$-QM rel $\partial G$, then $f$ is $\eta$-QS rel $\partial G$ with $\eta=\eta_{\theta, c}$. 
Proof. The proof of the absolute case in $R^{n}$, [V $\left.\ddot{a}_{1}, 3.14\right]$ needs only slight modifications. Write $z_{1}=x_{0}, M=d(G)=d(\partial G), M^{\prime}=d\left(G^{\prime}\right)=d\left(\partial G^{\prime}\right)$. Choose points $z_{2}, z_{3} \in \partial G$ with $\left|z_{2}-z_{3}\right| \geq M / 2$. It suffices to show that the conditions of Theorem 5.9 hold with some $\lambda=\lambda(\theta, c)$. Since

$$
\left|z_{1}-z_{2}\right| \geq \delta\left(x_{0}\right) \geq M / c, \quad\left|z_{1}-z_{3}\right| \geq M / c, \quad\left|z_{2}-z_{3}\right| \geq M / 2
$$

the first condition of 5.9 is true with $\lambda=c \vee 2$. For $j=2,3$ we have

$$
\left|f z_{j}-f z_{1}\right| \geq \delta^{\prime}\left(x_{0}^{\prime}\right) \geq M^{\prime} / c
$$

and it remains to find an upper bound for $M^{\prime} /\left|f z_{2}-f z_{3}\right|$.

Choose $z_{4} \in \partial G$ with $\left|f z_{4}-f z_{3}\right| \geq M^{\prime} / 3$. The quadruple $Q=\left(z_{2}, z_{1}, z_{3}, z_{4}\right)$ is in $(\bar{G}, \partial G)$, and $\tau(Q) \leq 2 c$. Since

$$
\tau(f Q) \geq \frac{M^{\prime}}{3 c\left|f z_{2}-f z_{3}\right|}
$$

and since $f$ is $\theta$-QM rel $\partial G$, we obtain

$$
\frac{M^{\prime}}{\left|f z_{2}-f z_{3}\right|} \leq 3 c \theta(2 c) \text {. 口 }
$$

\section{Uniform domains}

6.1. Introduction to Section 6 . Uniform domains in $R^{n}$ were introduced by Martio and Sarvas [MS] in 1979. A related concept was independently studied by Jones [Jo], and the equivalence of these two approaches was proved in [GO]. In this section we shall consider uniform domains in a Banach space. The definition will be given in terms of length cigars, and alternative characterizations are given in terms of the $\mathrm{QH}$ metric.

6.2. Cigars. Let $\gamma \subset E$ be an arc with endpoints $a, b$. For $x \in \gamma$ we set

$$
\varrho_{d}(x)=d(\gamma[a, x]) \wedge d(\gamma[x, b]) .
$$

If $\gamma$ is rectifiable, we also define the function

$$
\varrho_{l}(x)=l(\gamma[a, x]) \wedge l(\gamma[x, b]) .
$$

For $c \geq 1$, the sets

$$
\begin{aligned}
\operatorname{cig}_{d}(\gamma, c) & =\cup\left\{B\left(x, \varrho_{d}(x) / c\right): x \in \gamma \backslash\{a, b\}\right\}, \\
\operatorname{cig}_{l}(\gamma, c) & =\cup\left\{B\left(x, \varrho_{l}(x) / c\right): x \in \gamma \backslash\{a, b\}\right\}
\end{aligned}
$$

are the diameter $c$-cigar and the length $c$-cigar, respectively, with core $\gamma$. The length cigar is only defined for a rectifiable $\gamma$. 
6.3. Uniform domains. Let $c \geq 1$. A domain $G \subset E$ is a $c$-uniform domain if each pair $a, b \in G$ can be joined by a rectifiable arc $\gamma$ satisfying the following uniformity conditions:

(1) $\operatorname{cig}_{l}(\gamma, c) \subset G$,

(2) $l(\gamma) \leq c|a-b|$.

We call (1) the cigar condition and (2) the turning condition. Observe that (1) can be rewritten as

(1') $\varrho_{l}(x) \leq c \delta(x)$

for all $x \in \gamma$.

In $R^{n}$ one can also characterize the uniform domains by diameter cigars and the so-called distance cigars, cf. $[\mathrm{MS}]$ and $\left[\mathrm{Vä}_{2}\right]$. This is no longer true in the general case. For example, the broken tube of I.4.12 is not a uniform domain although one can show that there is $c>1$ such that each pair $a, b \in G$ can be joined by an arc $\gamma$ such that $\operatorname{cig}_{d}(\gamma, c) \subset G$ and $d(\gamma) \leq c|a-b|$.

We first give examples of uniform domains. A simple lemma is needed:

6.4. Lemma. If $y, z \in S(1)$, then $|y-z| \leq 2 d(y,[0, z])$.

Proof. Let $x \in[0, z]$ and set $\alpha=|y-z| / 2$. If $|x| \leq 1-\alpha$, then $|y-x| \geq$ $|y|-|x| \geq \alpha$. If $|x| \geq 1-\alpha$, then

$$
|y-x| \geq|y-z|-|z-x|=2 \alpha-(1-|x|) \geq \alpha \text {. }
$$

6.5. Theorem. For $x_{0} \in E$ and $r>0$, the domains $B\left(x_{0}, r\right), B\left(x_{0}, r\right) \backslash\left\{x_{0}\right\}$ and $E \backslash\left\{x_{0}\right\}$ are $c$-uniform with a universal $c$.

Proof. We may assume that $x_{0}=0$ and $r=1$. We first consider the domain $G=B(1) \backslash\{0\}$. Suppose that $a, b \in G, a \neq b,|a| \geq|b|$. We show that $a$ and $b$ can be joined by an arc $\gamma$ satisfying the uniformity conditions in $G$. Setting

$$
a_{0}=a /|a|, \quad b_{0}=b /|b|, \quad t=\left|a_{0}-b_{0}\right| / 4
$$

we have $0 \leq t \leq 1 / 2$. We consider two cases.

Case 1. $|b| \leq 1-t$. Set $a_{1}=|b| a_{0}$ and apply 2.2 to find an arc $\gamma_{1} \subset S(|b|)$ joining $a_{1}$ and $b$ with $l\left(\gamma_{1}\right) \leq 2\left|a_{1}-b\right|=8 t|b|$. We show that $\gamma=\gamma_{1} \cup\left[a_{1}, a\right]$ has the desired properties.

If $x \in \gamma_{1}$, then

$$
\varrho_{l}(x) \leq l\left(\gamma_{1}\right) \leq 8 t|b|, \quad \delta(x)=|b| \wedge(1-|b|) \geq|b| \wedge t,
$$

and hence $\varrho_{l}(x) \leq 8(t \vee|b|) \delta(x)<8 \delta(x)$. If $x \in\left[a_{1}, a\right] \cap B(1 / 2)$, then

$$
\varrho_{l}(x) \leq l\left(\gamma_{1}\right)+\left|x-a_{1}\right| \leq 8 t|b|+|x| \leq 5|x|=5 \delta(x) .
$$


If $x \in\left[a_{1}, a\right] \backslash B(1 / 2)$, then

$$
\varrho_{l}(x) \leq|a-x| \leq 1-|x|=\delta(x) .
$$

Hence the cigar condition holds with $c=8$.

If $\left|a_{1}-b\right| \leq 2\left|a-a_{1}\right|$, then

$$
l(\gamma) \leq 5\left|a-a_{1}\right|=5(|a|-|b|) \leq 5|a-b| .
$$

If $\left|a_{1}-b\right| \geq 2\left|a-a_{1}\right|$, then

$$
l(\gamma) \leq 5\left|a_{1}-b\right| / 2, \quad|a-b| \geq\left|a_{1}-b\right|-\left|a-a_{1}\right| \geq\left|a_{1}-b\right| / 2,
$$

and we obtain in both cases the turning condition with $c=5$.

Case 2. $|b| \geq 1-t$. Set $a_{1}=(1-t) a_{0}, b_{1}=(1-t) b_{0}$, and join the points $a_{1}, b_{1}$ with an arc $\gamma_{1} \subset S(1-t)$ with $l\left(\gamma_{1}\right) \leq 2\left|a_{1}-b_{1}\right|=8 t(1-t)<8 t$. We show that $\gamma=\left[a, a_{1}\right] \cup \gamma_{1} \cup\left[b_{1}, b\right]$ is the desired arc. If $x \in\left[a, a_{1}\right] \cup\left[b, b_{1}\right]$, then $\varrho_{l}(x)<\delta(x)$. If $x \in \gamma_{1}$, then

$$
\varrho_{l}(x) \leq\left|a-a_{1}\right|+l\left(\gamma_{1}\right) \leq t+8 t=9 \delta(x),
$$

and we obtain the cigar condition with $c=9$.

By 6.4 we have

$$
|a-b|=|a|\left|a_{0}-b /\right| a|| \geq 2|a| t \geq t,
$$

which yields the turning condition

$$
l(\gamma) \leq|a|-1+t+8 t+|b|-1+t<10 t \leq 10|a-b| .
$$

Hence the domain $B(1) \backslash\{0\}$ is 10-uniform. The case $G=E \backslash\left\{x_{0}\right\}$ follows immediately from this. For $G=B(1)$ it suffices to observe that the line segment $[0, x]$ satisfies for all $x \in G$ uniformity conditions with $c=1$. $\square$

6.6. Other examples. Suppose that $T$ is a closed affine proper subspace of $E$. If codim $T \geq 2, E \backslash T$ is a domain. If codim $T=1, E \backslash T$ consists of two domains called half spaces. All these domains are $c$-uniform with universal $c$. The case $T=\left\{x_{0}\right\}$ is contained in 6.5. The proof of the general case is contained in [Al]. In fact, $c$ can be chosen to be any number greater than 2 .

If $G$ and $D$ are $c$-uniform domains with $G \subset \bar{D}$, then $G \cap D$ is a $c_{1}$-uniform domain with $c_{1}=c_{1}(c)$. In the case $E=R^{n}$ this is essentially Theorem 5.4 of [Väㄱ. In the general case, the proof needs some modification; for example, the distance cigars must be replaced by length cigars. A detailed proof is in [Al]. A direct proof for the case $D=E \backslash\left\{x_{0}\right\}$ is sketched in 6.7 below.

More examples can be obtained by auxiliary maps. We show in 6.26 that if $G$ is $c$-uniform and $f: G \rightarrow G^{\prime} \eta$-QM, then $G^{\prime}$ is $c_{1}$-uniform with $c_{1}=c_{1}(c, \eta)$. 
6.7. Lemma. Suppose that $G$ is a $c$-uniform domain and that $x_{0} \in G$. Then $G_{0}=G \backslash\left\{x_{0}\right\}$ is $c_{0}$-uniform with $c_{0}=c_{0}(c)$.

Proof. We may assume that $x_{0}=0$ and $\delta\left(x_{0}\right)=1$. Let $a, b \in G_{0}, a \neq b$. We describe how to construct an arc $\gamma_{0}$ from $a$ to $b$ satisfying the uniformity conditions in $G_{0}$. We consider 3 cases.

Case 1. $|a|<1$ and $|b|<1$. This case follows from 6.5.

Case 2. $|a| \geq 1 / 2$ and $|b| \geq 1 / 2$. Join $a$ to $b$ with an arc $\gamma$ satisfying the uniformity conditions in $G$. If $0 \notin \operatorname{cig}_{l}(\gamma, 3 c)$, choose $\gamma_{0}=\gamma$. If $0 \in \operatorname{cig}_{l}(\gamma, 3 c)$ it is easy to see that $\gamma$ meets $S(1 / 2)$. Orient $\gamma$ from $a$ to $b$, and choose the first point $a_{1}$ and the last point $b_{1}$ of $\gamma$ in $S(1 / 2)$. Apply 2.2 to choose an arc $\alpha$ joining $a_{1}$ and $b_{1}$ in $S(1 / 2)$ with $l(\alpha) \leq 2\left|a_{1}-b_{1}\right|$. Then

$$
\gamma_{0}=\gamma\left[a, a_{1}\right] \cup \alpha \cup \gamma\left[b_{1}, b\right] .
$$

Case 3. $|a|<1 / 2$ and $|b| \geq 1$. Let $\gamma$ and $b_{1}$ be as in Case 2. We obtain $\gamma_{0}$ by replacing $\gamma\left[a, b_{1}\right]$ by the union of an arc in $S(|a|)$ and a radial segment from $S(|a|)$ to $S(1 / 2)$.

6.8. Other approaches to uniformity. For $x, y \in G \neq E$, the numbers

$$
r_{G}(x, y)=\frac{|x-y|}{\delta(x) \wedge \delta(y)}, \quad j_{G}(x, y)=\ln \left(1+r_{G}(x, y)\right)
$$

are the relative distance and the Jones distance between $x$ and $y$ in $G$, respectively. We shall often abbreviate $j=j_{G}, j^{\prime}=j_{G^{\prime}}$. Slightly different but essentially equivalent versions of $j_{G}$ have been considered by Jones [Jo] and by Gehring-Osgood [GO]; the present expression is due to Vuorinen [ $\left.\mathrm{Vu}_{1}\right]$. By I.2.2 we always have

$$
j_{G}(x, y) \leq k_{G}(x, y) \text {. }
$$

The uniform domains in $R^{n}$ can be characterized by inequalities in the opposite direction. Indeed, either of the conditions

$$
k_{G} \leq c j_{G}, \quad k_{G} \leq c j_{G}+d
$$

is quantitatively equivalent to $c$-uniformity; see [GO], $\left[\mathrm{Vu}_{1}, 2.50(2)\right]$ and $[\mathrm{Ge}$, Theorem 6]. A free version of this result is given in 6.16.

We also consider a generalization of the inequality $k_{G} \leq c j_{G}$, suggested by Vuorinen $\left[\mathrm{Vu}_{1}, 2.49\right]$. Let $\psi:[0, \infty) \rightarrow[0, \infty)$ be a homeomorphism. A domain $G \neq E$ is called quasihyperbolically $\psi$-uniform, or briefly QH $\psi$-uniform, if

$$
k_{G}(x, y) \leq \psi\left(r_{G}(x, y)\right)
$$


for all $x, y \in G$. For the function $\psi(t)=c \ln (1+t)$ this gives the inequality $k_{G} \leq c j_{G}$. A somewhat surprising fact is that for a large class of functions $\psi$, this is no generalization at all. More precisely, let us call a homeomorphism $\psi:[0, \infty) \rightarrow$ $[0, \infty)$ slow if $\psi(t) / t \rightarrow 0$ as $t \rightarrow \infty$. The function $\psi(t)=c \ln (1+t)$ is clearly slow. We shall prove that $\mathrm{QH} \psi$-uniformity with a slow $\psi$ quantitatively implies the condition $k_{G} \leq c j_{G}$.

We start with results dealing with coarse length in uniform domains. They are needed in the proof of 6.22, which is useful in Section 7. To prove the equivalences described above, we only need the case $h=0$ of these results. Recall that an arc is $(c, h)$-solid if it is $h$-coarsely $c$-quasiconvex in the $\mathrm{QH}$ metric of $G$. Roughly speaking, we show that a solid arc cannot travel long distances near the boundary of a uniform domain.

6.9. Lemma. Suppose that $G \neq E$ and that $\gamma$ is an arc in $G \cap(\partial G+\bar{B}(r))$ with endpoints $a_{0}, a_{1}$ such that $\delta\left(a_{0}\right) \wedge \delta\left(a_{1}\right) \geq r / c_{1}$. Suppose also that $G$ is $\mathrm{QH}$ $\psi$-uniform with a slow $\psi$.

(1) If $\gamma$ is $(c, h)$-solid, then $d(\gamma) \leq M_{1}\left(c, h, c_{1}, \psi\right) r$.

(2) If $\gamma$ is a $c$-neargeodesic, then $l(\gamma) \leq M_{1}\left(c, c_{1}, \psi\right) r$.

Proof. To prove (1) we set $t=d(\gamma) / r$ and look for an upper bound $t \leq M_{1}$. The solidity and uniformity conditions give

$$
l_{k}(\gamma, h) \leq c k\left(a_{0}, a_{1}\right) \leq c \psi\left(r_{G}\left(a_{0}, a_{1}\right)\right) \leq c \psi\left(c_{1} t\right) .
$$

If $c \psi\left(c_{1} t\right) \leq h$, we can choose $M_{1}=\psi^{-1}(h / c) / c_{1}$. If $c \psi\left(c_{1} t\right) \geq h$, then 4.5 gives $d(\gamma) \leq M(h) c \psi\left(c_{1} t\right) r$, and hence

$$
1 \leq M(h) c c_{1} \frac{\psi\left(c_{1} t\right)}{c_{1} t} .
$$

Since $\psi$ is slow, this yields the desired bound $t \leq M_{1}\left(c, h, c_{1}, \psi\right)$.

To prove (2) we set $t=l(\gamma) / r$. An easy modification of the argument above gives

$$
1 \leq c c_{1} \frac{\psi\left(c_{1} t\right)}{c_{1} t}
$$

and hence $t \leq M_{1}\left(c, c_{1}, \psi\right)$.

6.10. Lemma. Suppose that $G$ is a $\mathrm{QH} \psi$-uniform domain with a slow $\psi$. Suppose also that $\gamma$ is an arc in $G \cap(\partial G+\bar{B}(r))$.

(1) If $\gamma$ is $(c, h)$-solid, then $d(\gamma) \leq M_{2}(c, h, \psi) r$.

(2) If $\gamma$ is a $c$-neargeodesic, then $l(\gamma) \leq M_{2}(c, \psi) r$. 
Proof. Replacing $r$ by a smaller number we may assume that $\delta\left(a_{0}\right)>r / 2$ for some $a_{0} \in \gamma$. Dividing $\gamma$ to two subarcs we may further assume that $a_{0}$ is an endpoint of $\gamma$. Choose successive points $a_{1}, a_{2}, \ldots$ of $\gamma$ such that $a_{j}$ is the last point of $\gamma$ with $\delta\left(a_{j}\right) \geq 2^{-j} r$. The sequence $\left(a_{j}\right)$ may be finite or infinite. Set $\gamma_{j}=\gamma\left[a_{j-1}, a_{j}\right]$. In the part (1) we obtain from 6.9(1)

$$
d\left(\gamma_{j}\right) \leq M_{1}(c, h, 2, \psi) r / 2^{j-1},
$$

and hence $d(\gamma) \leq 2 M_{1} r$. The part (2) follows similarly from 6.9(2).

6.11. Lemma. For every slow $\psi$ and for all $c \geq 1, h \geq 0$ there is a number $q=q(c, h, \psi) \in(0,1)$ with the following property: Suppose that $G$ is a QH $\psi$-uniform domain and that $\gamma$ is a $(c, h)$-solid arc starting at $x_{0}$ and containing a point $x$ with $\delta(x) \leq q \delta\left(x_{0}\right)$. Then for $\gamma_{x}=\gamma \backslash \gamma\left[x_{0}, x\right)$ we have $d\left(\gamma_{x}\right) \leq M_{3}(c, h, \psi) \delta(x)$. If $h=0$, then $l\left(\gamma_{x}\right) \leq M_{3}(c, \psi) \delta(x)$.

Proof. Let $M_{2}=M_{2}(c, h, \psi)$ be the constant given by 6.10 . We show that one can choose

$$
q=\exp \left[-2\left(h \vee c \psi\left(M_{2}\right)\right)\right] .
$$

Let $\gamma, x_{0}, x$ satisfy the conditions of 6.11 with this $q$. Setting $r=\delta(x) / q$ we have $r \leq \delta\left(x_{0}\right)$. It suffices to show that $\gamma_{x} \subset \partial G+\bar{B}(r)$, since then 6.10 gives the result with $M_{3}=M_{2} / q$.

Assume that $\gamma_{x} \not \subset \partial G+\bar{B}(r)$. Let $x_{2}$ be the first point of $\gamma_{x}$ with $\delta\left(x_{2}\right)=r$. Since $\delta\left(x_{0}\right) \geq r$, we can choose the last point $x_{1}$ of $\gamma\left[x_{0}, x\right]$ with $\delta\left(x_{1}\right)=r$. Then for $\alpha=\gamma\left[x_{1}, x_{2}\right]$ we have

$$
l_{k}\left(\gamma\left[x_{1}, x\right], h\right) \leq l_{k}(\alpha, h) \leq c k\left(x_{1}, x_{2}\right) \leq c \psi\left(\left|x_{1}-x_{2}\right| / r\right) \leq c \psi(d(\alpha) / r) .
$$

By 6.10 we have $d(\alpha) \leq M_{2} r$. Using I.2.2 and 4.3(5) we obtain

$$
\ln \left(1+\frac{\left|x_{1}-x\right|}{q r}\right) \leq k\left(x_{1}, x\right) \leq k\left(\gamma\left[x_{1}, x\right]\right) \leq h \vee c \psi\left(M_{2}\right)=\frac{1}{2} \ln \frac{1}{q} .
$$

On the other hand, we have

$$
\left|x_{1}-x\right| \geq \delta\left(x_{1}\right)-\delta(x)=(1-q) r,
$$

which gives the contradiction

$$
\ln \left(1+\frac{\left|x_{1}-x\right|}{q r}\right) \geq \ln \frac{1}{q} \text {. }
$$

6.12. Theorem. Suppose that $G$ is a $\mathrm{QH} \psi$-uniform domain with a slow $\psi$ and that $\gamma \subset G$ is a $c$-neargeodesic with endpoints $a_{0}$ and $a_{1}$. Then $\gamma$ satisfies the uniformity conditions

(1) $\operatorname{cig}_{l}\left(\gamma, c_{1}\right) \subset G$,

(2) $l(\gamma) \leq c_{1}\left|a_{0}-a_{1}\right|$,

where $c_{1}$ depends only on $c$ and $\psi$. 
Proof. Choose $x_{0} \in \gamma$ such that $\delta\left(x_{0}\right)$ is maximal. Let $q=q(c, 0, \psi)$ be the number given by 6.11. If $x \in \gamma\left[a_{0}, x_{0}\right]$ and $\delta(x) \leq q \delta\left(x_{0}\right)$, then 6.11 implies

$$
l\left(\gamma\left[a_{0}, x\right]\right) \leq M_{3}(c, \psi) \delta(x) .
$$

If $x \in \gamma\left[a_{0}, x_{0}\right]$ and $\delta(x) \geq q \delta\left(x_{0}\right)$, then 6.10 with $r \mapsto \delta\left(x_{0}\right)$ yields

$$
l\left(\gamma\left[a_{0}, x\right]\right) \leq M_{2}(c, \psi) \delta\left(x_{0}\right) \leq\left(M_{2} / q\right) \delta(x) .
$$

Considering similarly the arc $\gamma\left[a_{1}, x\right]$ we conclude that (1) is true with $c_{1}(c, \psi)=$ $M_{3} \vee\left(M_{2} / q\right)$.

To prove (2) write $t=\left|a_{0}-a_{1}\right|$. We may assume that $\delta\left(a_{0}\right) \leq \delta\left(a_{1}\right)$. We consider two cases.

Case 1. $\delta\left(a_{0}\right) \leq t$. We may assume that $l(\gamma)>2 t$. Choose points $b_{0}$ and $b_{1}$ of $\gamma$ such that

$$
l\left(\gamma\left[a_{0}, b_{0}\right]\right)=t=l\left(\gamma\left[a_{1}, b_{1}\right]\right) .
$$

By (1) we have $t \leq c_{1} \delta\left(b_{0}\right)$ and $t \leq c_{1} \delta\left(b_{1}\right)$. Hence

$$
r_{G}\left(b_{0}, b_{1}\right) \leq \frac{\left|b_{0}-a_{0}\right|+\left|a_{0}-a_{1}\right|+\left|a_{1}-b_{1}\right|}{t / c_{1}} \leq 3 c_{1},
$$

and hence

$$
k\left(b_{0}, b_{1}\right) \leq \psi\left(3 c_{1}\right) .
$$

For each $x \in \gamma\left[b_{0}, b_{1}\right]$ we have

$$
k\left(x, b_{0}\right) \leq l_{k}\left(\gamma\left[b_{0}, x\right]\right) \leq l_{k}\left(\gamma\left[b_{0}, b_{1}\right]\right) \leq c k\left(b_{0}, b_{1}\right) \leq c \psi\left(3 c_{1}\right) .
$$

By I.2.2 this yields

$$
\left|x-b_{0}\right| \leq \delta\left(b_{0}\right)\left(e^{c \psi\left(3 c_{1}\right)}-1\right) .
$$

Since

$$
\delta\left(b_{0}\right) \leq \delta\left(a_{0}\right)+\left|a_{0}-b_{0}\right| \leq \delta\left(a_{0}\right)+t \leq 2 t
$$

we obtain

$$
\delta(x) \leq \delta\left(b_{0}\right)+\left|x-b_{0}\right| \leq 2 t e^{c \psi\left(3 c_{1}\right)}=M_{4}(c, \psi) t .
$$

Integration along $\gamma\left[b_{0}, b_{1}\right]$ gives

$$
c k\left(b_{0}, b_{1}\right) \geq l_{k}\left(\gamma\left[b_{0}, b_{1}\right]\right) \geq \frac{l\left(\gamma\left[b_{0}, b_{1}\right]\right)}{M_{4} t} .
$$

By (6.13) this implies

$$
l\left(\gamma\left[b_{0}, b_{1}\right]\right) \leq M_{5}(c, \psi) t .
$$

Hence (2) holds with $c_{1}$ replaced by $M_{5}+2$. 
Case 2. $\delta\left(a_{0}\right)=r \geq t$. This case makes no use of the QH uniformity of $G$. Since $\delta\left(a_{1}\right) \geq r$, we have $\delta(x) \geq r / 2$ for all $x \in\left[a_{0}, a_{1}\right]$. Integration along this line segment yields

$$
k\left(a_{0}, a_{1}\right) \leq 2 t / r .
$$

Let $\alpha:[0, \lambda] \rightarrow \gamma$ be the arc-length parametrization of $\gamma$ with $\lambda=l(\gamma)$ and $\alpha(0)=a_{0}$. Since

$$
\delta(\alpha(s)) \leq \delta\left(a_{0}\right)+\left|a_{0}-\alpha(s)\right| \leq r+s,
$$

we obtain

$$
l_{k}(\gamma)=\int_{0}^{\lambda} \frac{d s}{\delta(\alpha(s))} \geq \int_{0}^{\lambda} \frac{d s}{r+s}=\ln \left(1+\frac{\lambda}{r}\right) .
$$

Since $\gamma$ is a $c$-neargeodesic, this and (6.14) imply

$$
\ln \left(1+\frac{\lambda}{r}\right) \leq \frac{2 c t}{r}
$$

Setting $u=r / t$ we obtain

$$
\lambda / t=u\left(e^{2 c / u}-1\right) .
$$

Since $u \geq 1$ and since the right-hand side is bounded for $u \geq 1$, this implies (2).

6.15. Lemma. Suppose that $a, b \in G \neq E$. Then the following conditions are quantitatively equivalent:

(1) $k(a, b) \leq c j(a, b), c \geq 1$.

(2) $k(a, b) \leq c j(a, b)+\bar{d}, c \geq 1, d \geq 0$.

Proof. Trivially (1) implies (2). Assume that (2) holds, and set $r=r_{G}(a, b)$. Suppose first that $r \leq 1 / 2$. Since now $r \ln 2 \leq \ln (1+r)$, I.2.5 implies

$$
k(a, b) \leq 2 r \leq \frac{2}{\ln 2} j(a, b) .
$$

Next assume that $r \geq 1 / 2$. Then $j(a, b) \geq \ln (3 / 2)$, and hence

$$
\frac{k(a, b)}{j(a, b)} \leq c+\frac{d}{\ln (3 / 2)} \text {. }
$$

6.16. Theorem. For a domain $G \neq E$, the following conditions are quantitatively equivalent:

(1) $G$ is $c$-uniform,

(2) $k_{G} \leq c j_{G}$,

(3) $k_{G} \leq c j_{G}+d$,

(4) $G$ is QH $\psi$-uniform with a slow $\psi$. 
Proof. By $6.15,(3)$ implies (2). Since the function $\psi(t)=c \ln (1+t)$ is slow, (2) clearly implies (4). The implication $(4) \Rightarrow(1)$ follows from 6.12 and 3.3 . Finally, the implication $(1) \Rightarrow(3)$ can be proved with obvious modifications as in the case $E=R^{n}$ [GO, Theorem 1]. Observe that [GO] uses a slightly different version of the Jones distance, call it $j_{G}^{*}(a, b)$, but one has always $j_{G}^{*} \leq j_{G} \leq 2 j_{G}^{*}$. व

6.17. Remark. Inspection of the proofs shows that one can replace the slowness condition in 6.16 by the weaker condition

$$
\limsup _{t \rightarrow \infty} \frac{\psi(t)}{t}=v<1
$$

Indeed, assume that (4) of 6.16 holds with such $\psi$. Set $c=c_{1}=v^{-1 / 4}$. Thus $c$ and $c_{1}$ depend only on $\psi$. Since $c c_{1} v=v^{1 / 2}<1$, the proof of 6.9(2) is valid with these $c$ and $c_{1}$, and we get $M_{1}=M_{1}(\psi)$. In the proof of $6.10(2)$ we replace the conditions $\delta\left(a_{j}\right) \geq 2^{-j} r$ by $\delta\left(a_{j}\right) \geq c_{1}^{-j} r$ and obtain $l(\gamma) \leq M_{2} r$ with $M_{2}=M_{1}\left(1-c_{1}^{-1}\right)^{-1}=M_{2}(\psi)$. Then the case $h=0$ of 6.11 is also true with these $\psi$ and $c$ giving $q$ and $M_{3}$ depending on $\psi$. It follows that the proof of 6.12 is valid with these $\psi$ and $c$ giving a number $c_{1}=c_{1}(\psi)$, which should not be confused with the number $c_{1}=v^{-1 / 4}$ above. Since each pair of points in $G$ can be joined by a $c$-neargeodesic by $3.3, G$ is $c_{1}$-uniform.

The condition (6.18) is sharp in the sense that it cannot be replaced by $v \leq 1$. For example, each convex domain is $\mathrm{QH} \psi$-uniform with $\psi(t)=t$, but a parallel strip in $R^{2}$ is not a uniform domain.

From 6.12 and 6.16 we immediately get the following result, which in the case $E=R^{n}=E^{\prime}, c_{1}=1$ is given by [GO, Corollary 2, p. 59]:

6.19. Theorem. Suppose that $G \neq E$ is a $c$-uniform domain and that $\gamma$ is a $c_{1}$-neargeodesic in $G$ with endpoints $a_{0}, a_{1}$. Then there is $c_{2}=c_{2}\left(c, c_{1}\right) \geq 1$ such that

(1) $\operatorname{cig}_{l}\left(\gamma, c_{2}\right) \subset G$

(2) $l(\gamma) \leq c_{2}\left|a_{0}-a_{1}\right|$. व

6.20. Remark. Theorem 6.19 means that in a uniform domain, any neargeodesic is the core of a length cigar satisfying the uniformity conditions. We shall next prove a coarse version of this, replacing the neargeodesic by a solid arc and length by diameter. Both results will be needed in the proofs of 7.3 and 7.9. An auxiliary result is needed:

6.21. Lemma. Suppose that $G \neq E$ and that $\gamma$ is a $(c, h)$-solid arc in $G$ with endpoints $a_{0}, a_{1}$ such that $\delta\left(a_{0}\right) \wedge \delta\left(a_{1}\right)=r \geq\left|a_{0}-a_{1}\right|$. Then there is $c_{2}=c_{2}(c) \geq 1$ such that

$$
d(\gamma) \leq c_{2}\left|a_{0}-a_{1}\right| \vee 2 r\left(e^{h}-1\right)
$$


Proof. We may assume that $\delta\left(a_{1}\right) \geq \delta\left(a_{0}\right)=r$. Setting $t=\left|a_{0}-a_{1}\right|$ and integrating along $\left[a_{0}, a_{1}\right]$ we get $k\left(a_{0}, a_{1}\right) \leq 2 t / r$ as in the proof of (6.14). It suffices to find an estimate

$$
\left|x-a_{0}\right| \leq c_{2} t \vee r\left(e^{h}-1\right)
$$

for an arbitrary $x \in \gamma$.

If $k\left(x, a_{0}\right) \leq h$, then I.2.2 gives $\left|x-a_{0}\right| \leq r\left(e^{h}-1\right)$. Suppose that $k\left(x, a_{0}\right)$ $\geq h$. Choose successive points $a_{0}=x_{0}, \ldots, x_{n}=x$ of $\gamma, n \geq 1$, such that

$$
h \leq k\left(x_{j-1}, x_{j}\right) \leq 2 h
$$

for all $j$. Then

$$
n h \leq l_{k}(\gamma, h) \leq c k\left(a_{0}, a_{1}\right) \leq 2 c t / r .
$$

This implies $k\left(x, a_{0}\right) \leq 2 n h \leq 4 c t / r$, and hence

$$
\left|x-a_{0}\right| \leq \delta\left(a_{0}\right)\left(e^{k\left(x, a_{0}\right)}-1\right) \leq r\left(r^{4 c t / r}-1\right) .
$$

Setting $u=r / t$ we have $u \geq 1$ and

$$
\left|x-a_{0}\right| / t \leq u\left(e^{4 c / u}-1\right) .
$$

Since the right-hand side is bounded for $u \geq 1$, the lemma follows. ㅁ

6.22. Theorem. Suppose that $\gamma \subset G \neq E$ is a $(c, h)$-solid arc with endpoints $a_{0}, a_{1}$ and that $G$ is a $c_{1}$-uniform domain. Then there is $c_{2}=c_{2}\left(c, h, c_{1}\right)$ $\geq 1$ such that for $r=\delta\left(a_{0}\right) \wedge \delta\left(a_{1}\right)$ we have

(1) $\operatorname{cig}_{d}\left(\gamma, c_{2}\right) \subset G$,

(2) $d(\gamma) \leq c_{2}\left(\left|a_{0}-a_{1}\right| \vee 2 r\left(e^{h}-1\right)\right)$.

Proof. Choose $x_{0} \in \gamma$ for which $\delta\left(x_{0}\right)$ is maximal. For (1) it suffices to find $c_{2}=c_{2}\left(c, h, c_{1}\right)$ such that

$$
d\left(\gamma\left[a_{0}, x\right]\right) \leq c_{2} \delta(x)
$$

for all $x \in \gamma\left[a_{0}, x_{0}\right]$.

By $6.16 G$ is $\mathrm{QH} \psi$-uniform with a slow $\psi$ depending only on $c_{1}$. Let $q=q(c, h, \psi) \in(0,1)$ be the number given by 6.11 . If $\delta(x) \leq q \delta\left(x_{0}\right)$, then (6.23) follows from 6.11. If $\delta(x) \geq q \delta\left(x_{0}\right)$, we apply 6.10 with the substitution $r \mapsto \delta\left(x_{0}\right), \gamma \mapsto \gamma\left[a_{0}, x\right]$. We obtain

$$
d\left(\gamma\left[a_{0}, x\right]\right) \leq M_{2}(c, h, \psi) \delta\left(x_{0}\right),
$$

and hence (6.23) holds with $c_{2}=M_{2} / q$. 
To prove (2) set $t=\left|a_{0}-a_{1}\right|$. We may assume that $\delta\left(a_{1}\right) \geq \delta\left(a_{0}\right)=r$ and that $d(\gamma)>2 t$. If $r \geq t$, then (2) follows from 6.21. Suppose that $r \leq t$. Choose points $y_{0}, y_{1} \in \gamma$ such that $d\left(\gamma\left[a_{i}, y_{i}\right]\right)=t$ for $i=1,2$. By (1) we have

$$
\delta\left(y_{i}\right) \geq d\left(\gamma\left[a_{i}, y_{i}\right]\right) / c_{2}=t / c_{2}
$$

Since

$$
\left|y_{0}-y_{1}\right| \leq\left|y_{0}-a_{0}\right|+\left|a_{0}-a_{1}\right|+\left|a_{1}-y_{1}\right| \leq 3 t,
$$

and since $G$ is $\mathrm{QH} \psi$-uniform, this implies

$$
k\left(y_{0}, y_{1}\right) \leq \psi\left(r_{G}\left(y_{0}, y_{1}\right)\right) \leq \psi\left(3 c_{2}\right)
$$

By $4.3(5)$ and by the $(c, h)$-solidity of $\gamma$ we get

$$
k\left(\gamma\left[y_{0}, y_{1}\right]\right) \leq h \vee l_{k}\left(\gamma\left[y_{0}, y_{1}\right], h\right) \leq h \vee c k\left(y_{0}, y_{1}\right) \leq h \vee c \psi\left(3 c_{2}\right)=c_{3}\left(c, h, c_{1}\right)
$$

Hence $\gamma\left[y_{0}, y_{1}\right]$ is contained in the QH ball $B_{k}\left(y_{0}, c_{3}\right)$. By I.2.2 this implies that $\gamma \subset B\left(y_{0}, R\right)$ with

$$
R=\delta\left(y_{0}\right)\left(e^{c_{3}}-1\right)+t \leq\left(r+\left|a_{0}-y_{0}\right|\right)\left(e^{c_{3}}-1\right)+t \leq 2 t e^{c_{3}}-t .
$$

This implies (2) with $c_{2}$ replaced by $4 e^{c_{3}}-2$. $\square$

6.24. Quasimöbius invariance. In the basic paper [MS, 2.15], Martio and Sarvas proved that QC maps $f: R^{n} \rightarrow R^{n}$ preserve the class of uniform domains. A different proof was given in [GO, Corollary 3, p. 65]. More generally, uniformity is preserved by QM maps $f: G \rightarrow G^{\prime}$ of domains in $R^{n}$. This is obtained by modifying the proofs mentioned above or by using the characterization of uniform domains in terms of cross ratios, given by Martio [Ma]. The latter method was extended by the author [V $\left.\ddot{a}_{1}, 4.11\right]$ to a large class of spaces including all Banach spaces. However, the definition of a uniform domain in [Vä $\left.{ }_{1}\right]$ is not equivalent to the definition of the present paper in infinite-dimensional spaces. We shall next use the ideas of [GO] to prove the QM invariance of uniform domains in Banach spaces. For the notation of the following lemma, see 6.8 .

6.25. Lemma. Suppose that $G \neq E, G^{\prime} \neq E^{\prime}$ and that $f: G \rightarrow G^{\prime}$ is an $\eta$-QM homeomorphism. Then

$$
j^{\prime}(f a, f b) \leq M j(a, b)+C
$$

for all $a, b \in G$ with $M$ and $C$ depending only on $\eta$. 
Proof. We first consider two special cases.

Case 1. $0 \notin G$ and $f$ is the restriction of the inversion $u(x)=x /|x|^{2}$. Let $a, b \in G$ and set $r=r_{G}(a, b), r^{\prime}=r_{G^{\prime}}(u a, u b)$. We may assume that $\delta^{\prime}(u a) \leq \delta^{\prime}(u b)$. Choose $x \in \partial G$ such that $|u x-u a| \leq 2 \delta^{\prime}(u a)$. By [V $\ddot{a}_{1},(1.7)$ ] we obtain

$$
\frac{|u a-u b|}{\delta^{\prime}(u a)} \leq \frac{18|a-b||x|}{|x-a||b|} .
$$

Together with the inequalities

$$
|x| \leq|x-a|+|a-b|+|b|, \quad|x-a| \geq \delta(a), \quad|b| \geq \delta(b)
$$

this yields

$$
r^{\prime} \leq 18|a-b|\left(\frac{1}{\delta(b)}+\frac{|a-b|}{\delta(a) \delta(b)}+\frac{1}{\delta(a)}\right) \leq 18\left(2 r+r^{2}\right) .
$$

Hence $1+r^{\prime} \leq 18(1+r)^{2}$, and we obtain the lemma with $M=2, C=\ln 18$.

Case 2. $f$ is $\eta$-QS. Now $f$ extends to an $\eta$-QS homeomorphism $\bar{f}: \bar{G} \rightarrow \bar{G}^{\prime}$. Let $a, b \in G$, and choose $x \in \partial G$ with $|f a-\bar{f} x| \leq 2 \delta^{\prime}(f a)$. Setting $r=r_{G}(a, b)$ we obtain

$$
\frac{|f a-f b|}{\delta^{\prime}(f a)} \leq 2 \frac{|f a-f b|}{|f a-\bar{f} x|} \leq 2 \eta\left(\frac{|a-b|}{|a-x|}\right) \leq 2 \eta(r) .
$$

Since $G$ is connected, [ $\left.\mathrm{TV}_{1}, 3.12\right]$ implies that one can choose $\eta$ to be of the form $\eta(t)=C_{1}\left(t^{\alpha} \vee t^{1 / \alpha}\right), C_{1} \geq 1, \alpha \geq 1$. Since we may assume that $\delta^{\prime}(f a) \leq \delta^{\prime}(f b)$, we obtain

$$
r_{G^{\prime}}(f a, f b) \leq 2 C_{1}\left(r^{\alpha} \vee r^{1 / \alpha}\right) .
$$

If $r \leq 1$, then the lemma holds with $M=0, C=\ln \left(1+2 C_{1}\right)$. If $r \geq 1$, then $1+r^{\alpha} \leq(1+r)^{\alpha}$, and thus we can chose $M=\alpha, C=\ln 2 C_{1}$.

The general case is reduced to the special cases as follows: First extend $f$ to an $\eta$-QM embedding $\bar{f}: \bar{G} \rightarrow \dot{E}^{\prime}$ applying [Vä $1,3.19$ ], where the misprinted $f A$ should be replaced by $\overline{f A}$. By auxiliary translations we may assume that $0 \in \partial G$ and that $\bar{f}(0)$ is either 0 or $\infty$. Furthermore, we can use auxiliary inversions and Case 1 to normalize the map so that $\infty \in \partial G$ and $\bar{f}(\infty)=\infty$. Then $f$ is $\eta$-QS, and the result follows from Case 2.

6.26. Theorem. Suppose that $f: G \rightarrow G^{\prime}$ is an $\eta$-QM homeomorphism and that $G$ is a $c$-uniform domain. Then $G^{\prime}$ is $c_{1}$-uniform with $c_{1}=c_{1}(c, \eta)$.

Proof. If $G=E$, then $G^{\prime}=E^{\prime}$ by [Vä ${ }_{1}$, p. 226] or, alternatively, by I.5.13 and I.5.18. We may thus assume that $G \neq E, G^{\prime} \neq E^{\prime}$. By I.5.18 and by 4.14, there are $M \geq 1$ and $C \geq 0$ depending only on $\eta$ such that

$$
k^{\prime}(f a, f b) \leq M k(a, b)+C
$$


for all $a, b \in G$. Applying 6.25 to $f^{-1}$ we can write

$$
j(a, b) \leq M_{1} j^{\prime}(f a, f b)+C_{1}
$$

with $M_{1}, C_{1}$ depending only on $\eta$. By 6.16 , we have $k \leq c_{0} j$ with $c_{0}=c_{0}(c)$. These inequalities imply

$$
k^{\prime}(f a, f b) \leq M c_{0} j(a, b)+C \leq M M_{1} c_{0} j^{\prime}(f a, f b)+M c_{0} C_{1}+C,
$$

and the theorem follows from 6.16 . $\square$

6.27. Endcuts. Recall from 3.6 that an endcut of a domain $G$ is a half open arc $\gamma \subset G$ such that $\bar{\gamma}$ is a closed arc with one endpoint in $\partial G$. If $G \neq E$, the QH diameter $k(\gamma)$ of an endcut is always infinite. We next show that the converse is true for solid arcs in uniform domains:

6.28. Theorem. Suppose that $\gamma$ is a half open solid arc in a uniform domain $G \neq E$ and that $k(\gamma)=\infty$. Then $\gamma$ is an endcut of $G$. If $\gamma$ is also a neargeodesic, then either $\gamma$ converges to $\infty$ or $\gamma$ is rectifiable.

Proof. Assume that $G$ is $\mathrm{QH} \psi$-uniform with a slow $\psi$ and that $\gamma$ is $(c, h)$ solid in $G$. Since $k(\gamma)=\infty$, we have $l_{k}(\gamma, h)=\infty$. We may assume that $\gamma$ starts at the origin and that $\gamma$ does not converge to $\infty$. For $x \in \gamma$ write $\gamma_{x}=\gamma \backslash \gamma[0, x)$. There is $R>0$ such that $B(R)$ meets $\gamma_{x}$ for every $x \in \gamma$. If $z \in \gamma_{x} \cap B(R)$, then

$$
l_{k}(\gamma[0, z], h) \leq c k(0, z) \leq c \psi\left(\frac{R}{\delta(0) \wedge \delta(z)}\right)
$$

Since $l_{k}(\gamma, h)=\infty$, this implies $d(\gamma, \partial G)=0$.

Let $\varepsilon>0$. To prove that $\gamma$ is an endcut it suffices to find $x \in \gamma$ with $d\left(\gamma_{x}\right) \leq \varepsilon$. Let $q=q(c, h, \psi)$ and $M_{3}=M_{3}(c, h, \psi)$ be the numbers given by 6.11. Choose $x \in \gamma$ such that

$$
\delta(x) \leq q \delta(0) \wedge\left(\varepsilon / M_{3}\right)
$$

Then 6.11 gives $d\left(\gamma_{x}\right) \leq M_{3} \delta(x) \leq \varepsilon$.

In the case $h=0,6.11$ yields $l\left(\gamma_{x}\right) \leq M_{3} \delta(x)<\infty$ for any $x$ satisfying the inequality above. Hence $\gamma$ is rectifiable. $\square$ 


\section{Boundary behavior}

7.1. Introduction to Section 7. This section deals mainly with the boundary properties of homeomorphisms $f: G \rightarrow G^{\prime}$ between uniform domains. We show that if $f$ is CQH, it can be extended to a homeomorphism $\bar{f}: \bar{G} \rightarrow \bar{G}^{\prime}$, which is $\mathrm{QM}$ rel $\partial G$ in the norm metric. In the special case where $f$ is FQC, $\bar{f}$ is $\mathrm{QM}$ in the whole $\bar{G}$. These results are quantitative.

Many of the results are new also in the classical case $E=E^{\prime}=R^{n}$. In the case where $G$ and $G^{\prime}$ are half spaces of $R^{n}$, V.A. Efremovich and E.S. Tihomirova [ET] proved in 1964 that a solid map $f: G \rightarrow G^{\prime}$ extends to a homeomorphism $\bar{f}: \bar{G} \rightarrow \bar{G}^{\prime}$, and D.A. De-Spiller [DS] proved in 1970 that the induced map $\partial G \rightarrow$ $\partial G^{\prime}$ is quasiconformal. De-Spiller also proved that conversely, each quasiconformal map of $R^{n-1}$ can be extended to a homeomorphism of $R^{n-1} \times[0, \infty)$ which is solid in the open half space. We shall construct the corresponding extension from a Banach space $E$ to $E \times[0, \infty)$ in 7.26. A reflection principle is given in 7.35.

7.2. Terminology. Suppose that $G$ is a domain in $E$ and that $f: G \rightarrow E^{\prime}$ is a map. The cluster set of $f$ at a point $x_{0} \in \partial G$ is defined as

$$
\operatorname{clus}\left(f, x_{0}\right)=\cap \operatorname{cl} f[U \cap G]
$$

over all neighborhoods $U$ of $x_{0}$ in $\dot{E}$. Equivalently, a point $y \in \dot{E}^{\prime}$ belongs to clus $\left(f, x_{0}\right)$ if and only if there is a sequence of points $x_{j} \in G$ such that $x_{j} \rightarrow x_{0}$ and $f x_{j} \rightarrow y$.

In the general case, the cluster set may be empty even if $f$ is an $\mathrm{FQC}$ map onto a domain $G^{\prime}$; see I.4.12.

We next prove the crucial lemma of the paper. Its proof makes effective use of the theory of the preceding sections: the existence of neargeodesics, the CQH invariance of solid arcs, and the length cigar and diameter cigar theorems for uniform domains.

7.3. Fundamental lemma. Suppose that $G \neq E$ and $G^{\prime} \neq E^{\prime}$ are unbounded c-uniform domains and that $f: G \rightarrow G^{\prime}$ is $(M, C)$-CQH with $\infty \in$ clus $(f, \infty)$. Let $x, a, b$ be points in $G$ such that $|a-x| \leq|b-x|$ and $k(b, x) \geq$ $2 C \vee 1 / 2$. Then

$$
|f a-f x| \leq H|f b-f x|
$$

with $H=H(M, C, c)$.

Proof. By auxiliary similarities we normalize the situation so that $x=0$, $f x=0,|b|=1=|f b|$. Then $|a| \leq 1$ and we must find an upper bound $|f a| \leq H$ with $H$ depending only on $v=(M, C, c)$.

We first show that

$$
\delta^{\prime}(0) \leq 8 M
$$


Since this is trivial if $\delta^{\prime}(0) \leq 2$, we may assume that $\delta^{\prime}(0) \geq 2$. Then I.2.5 gives

$$
k^{\prime}(0, f b) \leq 2|f b| / \delta^{\prime}(0)=2 / \delta^{\prime}(0) .
$$

If $C \leq 1 / 4$, we have

$$
k^{\prime}(0, f b) \geq(k(0, b)-C) / M \geq 1 / 4 M
$$

If $C \geq 1 / 4$, then $k(0, b) \geq 2 C$, and hence

$$
k^{\prime}(0, f b) \geq C / M \geq 1 / 4 M
$$

which thus holds in all cases. By (7.5) this gives (7.4).

In what follows, we let $c_{1}, c_{2}, \ldots$ denote constants depending only on $v$. Applying 6.19 we first choose $c_{1} \geq 1$ such that the uniformity conditions hold with this $c_{1}$ for every 2-neargeodesic in $G$ and in $G^{\prime}$. Since $\infty \in \operatorname{clus}(f, \infty)$, we can choose $y \in G$ such that $|y| \geq 2$ and $|f y| \geq|f a|$. By 3.3 we can choose a 2neargeodesic $\alpha^{\prime}$ in $G^{\prime}$ joining $f a$ and $f y$. Let $z$ be a point of $\alpha^{\prime}$ with minimal norm. Then

$$
|z-f a| \wedge|z-f y| \geq|f a|-|z| .
$$

Since $\operatorname{cig}_{l}\left(\alpha^{\prime}, c_{1}\right) \subset G^{\prime}$, we obtain

$$
c_{1} \delta^{\prime}(z) \geq|f a|-|z| .
$$

Since (7.4) gives

$$
\delta^{\prime}(z) \leq \delta^{\prime}(0)+|z| \leq 8 M+|z|
$$

this implies

$$
2 c_{1}|z| \geq|f a|-8 c_{1} M .
$$

We may assume that $|f a| \geq 16 c_{1} M$, since otherwise there is nothing to prove. Then $2 c_{1}|z| \geq|f a| / 2$, and thus

$$
|f a| \leq 4 c_{1} d\left(0, \alpha^{\prime}\right)
$$

Choose a 2-neargeodesic $\beta^{\prime}$ in $G^{\prime}$ joining 0 and $f b$. By 4.15, the $\operatorname{arcs} \alpha=$ $f^{-1} \alpha^{\prime}$ and $\beta=f^{-1} \beta^{\prime}$ are $\left(c_{2}, h\right)$-solid in $G$ with $h=h(M, C)$. Hence, by 6.22 , there is $c_{3} \geq 1$ with

$$
\operatorname{cig}_{d}\left(\alpha, c_{3}\right) \cup \operatorname{cig}_{d}\left(\beta, c_{3}\right) \subset G .
$$

Choose points $a_{0} \in \alpha$ and $b_{0} \in \beta$ such that $\left|a_{0}\right|=3 / 2$ and $\left|b_{0}\right|=1 / 2$. Then

$$
\delta\left(a_{0}\right) \wedge \delta\left(b_{0}\right) \geq 1 / 2 c_{3} .
$$


Applying once more 3.3 we join $a_{0}$ and $b_{0}$ with a 2-neargeodesic $\gamma$ in $G$. Then $\operatorname{cig}_{l}\left(\gamma, c_{1}\right) \subset G$ and

$$
l(\gamma) \leq c_{1}\left|a_{0}-b_{0}\right| \leq 2 c_{1} .
$$

For every $x \in \gamma$ we have $\delta(x) \geq 1 / 4 c_{1} c_{3}$. Indeed, if $\left|x-a_{0}\right| \wedge\left|x-b_{0}\right|<1 / 4 c_{3}$, this follows from (7.7), and otherwise from the condition $\operatorname{cig}_{l}\left(\gamma, c_{1}\right) \subset G$. Hence

$$
l_{k}(\gamma) \leq 4 c_{1} c_{3} l(\gamma) \leq 8 c_{1}^{2} c_{3}=c_{4} .
$$

This implies $k\left(a_{0}, b_{0}\right) \leq c_{4}$ and hence

$$
k^{\prime}\left(f a_{0}, f b_{0}\right) \leq M c_{4}+C=c_{5} .
$$

Next observe that

$$
\left|f b_{0}\right| \leq l\left(\beta^{\prime}\right) \leq c_{1}|f b|=c_{1} .
$$

By (7.4) this implies

$$
\delta^{\prime}\left(f b_{0}\right) \leq \delta^{\prime}(0)+\left|f b_{0}\right| \leq 8 M+c_{1}=c_{6} .
$$

By I.2.2 these estimates yield

$$
\left|f a_{0}-f b_{0}\right| \leq \delta^{\prime}\left(f b_{0}\right) e^{k^{\prime}\left(f a_{0}, f b_{0}\right)} \leq c_{6} e^{c_{5}}=c_{7} .
$$

Together with (7.6) and (7.8) this gives the desired bound

$$
|f a| \leq 4 c_{1}\left|f a_{0}\right| \leq 4 c_{1}\left(c_{1}+c_{7}\right)=H(v) \text {. 口 }
$$

7.9. Theorem. Suppose that $G \neq E$ and $G^{\prime} \neq E^{\prime}$ are $c$-uniform domains and that $f: G \rightarrow G^{\prime}$ is $(M, C)$-CQH. Then $f$ extends to a homeomorphism $\bar{f}: \bar{G} \rightarrow \bar{G}^{\prime}$, and $\bar{f}$ is $\theta$-QM rel $\partial G$ with $\theta$ depending only on $(M, C, c)$. In particular, $\bar{f} \mid \partial G$ is $\theta-\mathrm{QM}$.

Proof. In the first part of the theorem, it suffices to show that $f$ has a limit at every point $x_{0} \in \partial G$. Indeed, then $f$ has a continuous extension $\bar{f}: \bar{G} \rightarrow \bar{G}^{\prime}$. By symmetry, $g=f^{-1}$ extends to a continuous map $\bar{g}: \bar{G}^{\prime} \rightarrow \bar{G}$. Then clearly $\bar{g} \bar{f}$ and $\bar{f} \bar{g}$ are identity maps, and thus $\bar{f}$ is a homeomorphism.

Performing an auxiliary inversion and recalling 6.26 and I.4.9, we may assume that $x_{0} \neq \infty$. Suppose that $f$ has no limit at $x_{0}$. By auxiliary similarities we may assume that $0 \in G, f(0)=0$, and $\delta(0)=1=\delta^{\prime}(0)$. For $r>0$ we set

$$
D(r)=f\left[G \cap B\left(x_{0}, r\right)\right] .
$$


There is $\alpha>0$ such that $d(D(r))>\alpha$ for all $r>0$, since otherwise $f$ has a limit at $x_{0}$ by the completeness of $E^{\prime}$. Since $\infty$ is not a limit, there is $R \geq \alpha$ such that the ball $B(R)$ meets $D(r)$ for all $r>0$.

Let $r>0$ and choose points $x, y \in G \cap B\left(x_{0}, r\right)$ such that $|f x|<R$ and $|f x-f y|>\alpha / 2$. Join $x$ and $y$ by a 2-neargeodesic $\gamma$ in $G$. By $4.15, f \gamma$ is $\left(c_{1}, h\right)$-solid in $G^{\prime}$ with $\left(c_{1}, h\right)$ depending only on $(M, C)$. In what follows, we let $c_{2}, c_{3}, \ldots$ denote constants depending only on $(M, C, c, \alpha, R)$. We have $\operatorname{cig}_{d}\left(f \gamma, c_{2}\right) \subset G^{\prime}$ by 6.22 . Choose $z \in \gamma$ with $|f z-f x|=\alpha / 4$. Then $|f z-f y| \geq$ $\alpha / 4$, and hence

$$
\delta^{\prime}(f z) \geq \alpha / 4 c_{2} .
$$

Join 0 and $f z$ by a 2 -neargeodesic $\beta^{\prime}$ in $G^{\prime}$. Then 6.19 gives $c_{3}$ with

$$
\operatorname{cig}_{l}\left(\beta^{\prime}, c_{3}\right) \subset G^{\prime}, \quad l\left(\beta^{\prime}\right) \leq c_{3}|f z| .
$$

Since $\delta^{\prime}(0)=1$, this and (7.10) give a lower bound $\delta^{\prime}(w) \geq 1 / c_{4}$ for all $w \in \beta^{\prime}$. Hence

$$
k^{\prime}(0, f z) \leq l_{k}\left(\beta^{\prime}\right) \leq c_{4} l\left(\beta^{\prime}\right) \leq c_{4} c_{3}|f z| .
$$

Here

$$
|f z| \leq|f z-f x|+|f x| \leq \alpha / 4+R<2 R .
$$

Since $f$ is $(M, C)-\mathrm{CQH}$, we obtain

$$
k(0, z) \leq 2 M R c_{4} c_{3}+C=c_{5} .
$$

On the other hand, 6.19 gives

$$
l(\gamma) \leq c_{3}|x-y| \leq 2 c_{3} r
$$

Hence

$$
\delta(z) \leq\left|z-x_{0}\right| \leq|z-x|+\left|x-x_{0}\right| \leq l(\gamma)+r \leq\left(2 c_{3}+1\right) r=c_{6} r .
$$

By I.2.2 this implies

$$
k(0, z) \geq \ln \frac{\delta(0)}{\delta(z)} \geq \ln \frac{1}{c_{6} r} .
$$

In view of (7.11), this gives a contradiction for small $r$. Hence $f$ has a homeomorphic extension $\bar{f}: \bar{G} \rightarrow \bar{G}^{\prime}$.

To prove the second part of the theorem, let $Q=(a, b, c, d)$ be a quadruple in $\bar{G}$ with $a, d \in \partial G$. Since $f^{-1}$ satisfies the same conditions as $f$, it follows from 5.8 that it suffices to find an estimate

$$
\tau(f Q) \leq \eta(\tau(Q))
$$


for some increasing $\eta:[0, \infty) \rightarrow[0, \infty)$ depending only on $v=(M, C, c)$. Performing auxiliary inversions we may assume that $d=\infty$ and $\bar{f} d=\infty$. Choose sequences $\left(a_{n}\right),\left(b_{n}\right),\left(c_{n}\right)$ in $G$ converging to $a, b, c$, respectively, such that the points $a_{n}, b_{n}, c_{n}$ are distinct for each $n$. Set $T_{n}=\left(a_{n}, b_{n}, c_{n}\right)$. Since $\rho\left(T_{n}\right)=$ $\left|a_{n}-b_{n}\right| /\left|a_{n}-c_{n}\right| \rightarrow \tau(Q)$ and $\rho\left(f T_{n}\right) \rightarrow \tau(f Q)$, it suffices to find an estimate

$$
\rho\left(f T_{n}\right) \leq \eta\left(\rho\left(T_{n}\right)\right)
$$

with $\eta=\eta_{v}$. Setting $\lambda=2 C \vee 1 / 2$ and observing that $\delta\left(a_{n}\right) \rightarrow 0$ we can assume that

$$
\left|c_{n}-a_{n}\right| \geq e^{\lambda} \delta\left(a_{n}\right)
$$

for each $n$.

Fix $n$ and choose an arc $\gamma$ joining $a_{n}$ and $b_{n}$ in $G$ with $l(\gamma) \leq c\left|a_{n}-b_{n}\right|$. Orient $\gamma$ so that $a_{n}$ is the first point. Set $y_{0}=a_{n}$, and let $y_{1}$ be the last point of $\gamma$ with $\left|y_{1}-y_{0}\right| \leq\left|c_{n}-y_{0}\right|$. Proceeding inductively, we let $y_{j+1}$ be the last point of $\gamma$ with $\left|y_{j+1}-y_{j}\right| \leq\left|y_{j}-y_{0}\right|$, and we stop as soon as we obtain $y_{s}$ with $y_{s}=b_{n}$. The process is finite, since $\gamma$ is compact and since $\left|y_{j}-y_{j-1}\right| \geq\left|c_{n}-y_{0}\right|$ for all $j \leq s-1$. Assume that $s \geq 2$. For $1 \leq j \leq s-1$ we have

$$
\left|y_{j}-a_{n}\right| \geq\left|y_{1}-a_{n}\right|=\left|c_{n}-a_{n}\right| \text {. }
$$

By (7.13) and by I.2.2, this implies that $k\left(y_{j}, a_{n}\right) \geq \lambda$ and $k\left(c_{n}, a_{n}\right) \geq \lambda$. By the Fundamental lemma 7.3, there is $H=H(v) \geq 1$ such that

$$
\left|f y_{1}-f a_{n}\right| \leq H\left|f c_{n}-f a_{n}\right|
$$

and

$$
\left|f y_{j+1}-f y_{j}\right| \leq H\left|f y_{j}-f a_{n}\right|
$$

for $1 \leq j \leq s-1$. These inequalities imply

$$
\left|f y_{j+1}-f a_{n}\right| \leq(1+H)\left|f y_{j}-f a_{n}\right| \leq(1+H)^{j}\left|f y_{1}-f a_{n}\right| \leq H(1+H)^{j}\left|f c_{n}-f a_{n}\right|
$$

for $0 \leq j \leq s-1$, and hence

$$
\left|f b_{n}-f a_{n}\right| \leq H(1+H)^{s-1}\left|f c_{n}-f a_{n}\right| .
$$

Clearly this is also true if $s=1$. Since

$$
c\left|a_{n}-b_{n}\right| \geq l(\gamma) \geq \sum_{j=1}^{s}\left|y_{j}-y_{j-1}\right| \geq(s-1)\left|c_{n}-a_{n}\right|,
$$

we have $s-1 \leq c \rho\left(T_{n}\right)$. Hence (7.12) is true with $\eta(t)=H(1+H)^{c t}$. व 
7.14. Theorem. Suppose that $G$ and $G^{\prime}$ are bounded $c$-uniform domains and that $c_{0} \geq 1, x_{0} \in G, x_{0}^{\prime} \in G^{\prime}$ with $d(G) \leq c_{0} \delta\left(x_{0}\right), d\left(G^{\prime}\right) \leq c_{0} \delta^{\prime}\left(x_{0}^{\prime}\right)$. Suppose also that $f: G \rightarrow G^{\prime}$ is an $(M, C)-\mathrm{CQH}$ map with $f x_{0}=x_{0}^{\prime}$. Then $f$ extends to a homeomorphism $\bar{f}: \bar{G} \rightarrow \bar{G}^{\prime}$, and $\bar{f}$ is $\eta$-QS rel $\partial G$ with $\eta$ depending only on $\left(M, C, c, c_{0}\right)$.

Proof. This follows directly from 7.9 and 5.11. $\square$

7.15. Theorem. Suppose that $f: B(1) \rightarrow B(1)$ is an $(M, C)-\mathrm{CQH}$ map between the unit balls of $E$ and $E^{\prime}$, and that $f(0)=0$. Then $f$ extends to a homeomorphism $\bar{f}: \bar{B}(1) \rightarrow \bar{B}(1)$, and $\bar{f}$ is $\eta$-QS rel $\partial B(1)$ with $\eta$ depending only on $(M, C)$.

Proof. Since a ball is $c$-uniform with a universal $c$ by 6.5 , the theorem is a corollary of 7.14. व

7.16. Theorem. Suppose that $G$ and $G^{\prime}$ are $c$-uniform domains and that $f: G \rightarrow G^{\prime}$ is $\varphi$-FQC. Then $f$ is $\eta$-QM with $\eta$ depending only on $\varphi$ and $c$.

Proof. If $G=E$ or $G^{\prime}=E^{\prime}$, the result follows from I.5.13. Suppose that $G \neq E, G^{\prime} \neq E^{\prime}$. By $7.9, f$ has a homeomorphic extension $\bar{f}: \bar{G} \rightarrow \bar{G}^{\prime}$. In view of 6.26 , we can use auxiliary inversions to normalize the situation so that $\infty \in \partial G$ and $\bar{f}(\infty)=\infty$. We show that $f$ is $\eta$-QS with $\eta=\eta_{\varphi, c}$. Now $G$ and $G^{\prime}$ are $c$-quasiconvex. By I.5.5, it suffices to show that $f$ is weakly $H$-QS with $H=H(\varphi, c)$.

Let $x, a, b \in G$ with $|a-x| \leq|b-x|$. By $4.14, f$ is $(M, 1 / 4)$-CQH with $M$ depending only on $\varphi$. By 7.3 , the desired inequality

$$
|f a-f x| \leq H|f b-f x|
$$

holds with $H=H(\varphi, c)$ provided that $k(b, x) \geq 1 / 2$. Suppose that $k(b, x)<1 / 2$. Then I.2.2 gives

$$
|b-x| \leq \lambda \delta(x), \quad \lambda=e^{1 / 2}-1<1 .
$$

Hence $a$ and $b$ are in the ball $B=B(x, \lambda \delta(x))$. By I.5.10, $f \mid B$ is $\eta$-QS with $\eta=\eta_{\varphi}$. Thus (7.17) holds with $H=\eta(1)$. $\square$

7.18. Theorem. Suppose that $G$ is a $c$-uniform domain and that $f: G \rightarrow G^{\prime}$ is $\varphi$-FQC. Then the following conditions are quantitatively equivalent:

(1) $G^{\prime}$ is $c_{1}$-uniform,

(2) $f$ is $\eta$-quasimöbius.

Proof. This follows from 7.16 and 6.26 . $\square$

7.19. Question. Does 7.18 remain true if $\varphi-\mathrm{FQC}$ is replaced by $(M, C)-\mathrm{CQH}$ and $\eta$-QM by $\eta$-QM rel $\partial G$ ? 
7.20. Remarks. In Theorem 7.9 we showed that for maps between uniform domains, CQH quantitatively implies QM rel $\partial G$. The uniformity condition can hardly be weakened. For example, conformal maps between non-uniform planar domains may have rather bad boundary behavior.

We next turn to the converse of 7.9 and show in 7.22 that for maps between uniform domains, QM rel $\partial G$ quantitatively implies $\mathrm{CQH}$. Here the uniformity plays a less important role. In fact, we shall prove the result for domains which are only $\mathrm{QH} \psi$-uniform. Recall from 6.8 that $G$ is $\mathrm{QH} \psi$-uniform if $\psi:[0, \infty) \rightarrow$ $[0, \infty)$ is a homeomorphism and if

$$
k_{G}(a, b) \leq \psi\left(r_{G}(a, b)\right)
$$

for all $a, b \in G$; here $r_{G}(a, b)=|a-b| /(\delta(x) \wedge \delta(y))$ is the relative distance between $a$ and $b$ in $G$. For example, all convex domains are QH $\psi$-uniform with $\psi(t)=t$.

In $\left[V \ddot{a}_{5}\right]$ we shall prove the result for a larger class of domains including all domains in $R^{n}$.

7.21. Lemma. Suppose that $0 \notin G$ and that $G$ is $\mathrm{QH} \psi$-uniform. Let $u$ be the inversion $u(x)=x /|x|^{2}$. Then $u G$ is $\mathrm{QH} \psi_{1}$-uniform with $\psi_{1}$ depending only on $\psi$.

Proof. Let $a, b \in G$ and set $r=r_{G}(a, b), r^{\prime}=r_{G^{\prime}}(u a, u b)$, where $G^{\prime}=u G$. Applying Case 1 of the proof of 6.25 to the inverse map $G^{\prime} \rightarrow G$ we obtain $r \leq 18\left(2 r^{\prime}+r^{2}\right)$. By I.4.9, $u$ is fully $36-\mathrm{QH}$. Thus

$$
k^{\prime}(u a, u b) \leq 36 k(a, b) \leq 36 \psi(r) \leq 36 \psi\left(18\left(2 r^{\prime}+r^{\prime 2}\right)\right)=\psi_{1}\left(r^{\prime}\right) \text {. }
$$

7.22. Theorem. Suppose that $G$ and $G^{\prime}$ are $\mathrm{QH} \psi$-uniform domains and that $f: \bar{G} \rightarrow \bar{G}^{\prime}$ is a homeomorphism with $f G=G^{\prime}$ such that $f$ is $\eta$-QM rel $\partial G$. Then $f \mid G$ is $(M, C)-\mathrm{CQH}$ with $(M, C)$ depending only on $\eta$ and $\psi$.

Proof. Performing auxiliary similarities we may assume that $0 \in \partial G$ and that $f(0)$ is either 0 or $\infty$. In view of 7.21 , we can use auxiliary inversions to normalize the map so that $\infty \in \partial G$ and $f(\infty)=\infty$. Then $f$ is $\eta$-QS rel $\partial G$.

Suppose that $a, b \in G$ with $k(a, b) \leq 1$. By 4.8 , it suffices to find an estimate

$$
k^{\prime}(f a, f b) \leq M_{0}
$$

with $M_{0}=M_{0}(\eta, \psi)$. We may assume that $\delta^{\prime}(f b) \leq \delta^{\prime}(f a)$. Choose $x \in \partial G$ with $|f x-f b| \leq 2 \delta^{\prime}(f b)$. Then

$$
r_{G^{\prime}}(f a, f b)=\frac{|f a-f b|}{\delta^{\prime}(f b)} \leq 2 \frac{|f a-f x|}{|f b-f x|}+2 \leq 2 \eta\left(\frac{|a-x|}{|b-x|}\right)+2 .
$$


By I.2.2 we have

$$
\frac{|a-b|}{\delta(b)} \leq e^{k(a, b)} \leq e
$$

Hence

$$
\frac{|a-x|}{|b-x|} \leq \frac{|a-b|+|b-x|}{|b-x|} \leq \frac{|a-b|}{\delta(b)}+1 \leq e+1<4 .
$$

By (7.24) this implies

$$
r_{G^{\prime}}(f a, f b) \leq 2 \eta(4)+2,
$$

which gives (7.23) with $M_{0}=\psi(2 \eta(4)+2)$. 口

7.25. Extension to a half space. For a Banach space $E$, we consider the space $E_{1}=E \times R^{1}$ as a Banach space with the norm $|(x, t)|=|x| \vee|t|$, and we identify $E$ with the subspace $E \times\{0\}$ of $E_{1}$. Let $H$ be the half space $E \times(0, \infty)$. Then $\partial H=\dot{E}=E \cup\{\infty\}$. For another Banach space $E^{\prime}$ we similarly define $E_{1}^{\prime}$ and $H^{\prime}$. The half spaces $H$ and $H^{\prime}$ are $c_{0}$-uniform domains with a universal $c_{0}$ by 6.6. Suppose that $F: H \rightarrow H^{\prime}$ is $\varphi$-solid. Then $F$ extends to a homeomorphism $\bar{F}: \bar{H} \rightarrow \bar{H}^{\prime}$ and induces an $\eta$-QM homeomorphism $f: \dot{E} \rightarrow \dot{E}^{\prime}$ with $\eta=\eta_{\varphi}$ by 7.9 .

We shall next show that conversely, every $\eta$-QM homeomorphism $f: \dot{E} \rightarrow \dot{E}^{\prime}$ can be extended to a homeomorphism $\bar{F}: \bar{H} \rightarrow \bar{H}^{\prime}$ such that the induced map $F: H \rightarrow H$ is $\varphi$-solid with $\varphi=\varphi_{\eta}$. In the case $E=R^{n}=E^{\prime}$, this was proved by De-Spiller [DS] in 1970. In [TV 2 ] we proved the stronger result that $F$ can be chosen to be $\mathrm{QH}$ and hence $\mathrm{QC}$ in $H^{n+1}=R^{n} \times(0, \infty)$. We do not know whether this is true in the general case.

The construction of $F$ in the proof of 7.26 was used as a preliminary step in $\left[\mathrm{TV}_{2}\right]$, and the solidity of $F$ in the euclidean case can be proved by compact families of embeddings; see [ $\left.\mathrm{TV}_{3}, 6.17\right]$ and $\left[\mathrm{TV}_{2}, 2.13\right]$. We shall give an elementary but somewhat lengthy direct proof.

7.26. Theorem. Suppose that $f: \dot{E} \rightarrow \dot{E}^{\prime}$ is $\eta$-QM. Then, with the notation of 7.25 , there is an extension of $f$ to a homeomorphism $\bar{F}: \bar{H} \rightarrow \bar{H}^{\prime}$ such that $F: H \rightarrow H^{\prime}$ is $\varphi$-solid with $\varphi=\varphi_{\eta}$.

Proof. By an auxiliary inversion we may assume that $f(\infty)=\infty$. Then $f$ is $\eta$-QS in $E$. For $x \in E$ and $t \geq 0$ we set

$$
\begin{aligned}
\tau(x, t) & =\sup \{|f y-f x|:|y-x| \leq t\}, \\
\bar{F}(x, t) & =(f x, \tau(x, t)) .
\end{aligned}
$$

Setting $\bar{F}(\infty)=\infty$ we obtain an extension $\bar{F}: \bar{H} \rightarrow \bar{H}^{\prime}$ of $f$ and its restriction $F: H \rightarrow H^{\prime}$. We show that $\bar{F}$ is the desired map. 
We first show that there are $s_{0}=s_{0}(\eta) \in(0,1 / 2]$ and embeddings $\mu, \theta:\left[0, s_{0}\right]$ $\rightarrow[0, \infty)$ with $\mu(0)=\theta(0)=0$ depending only on $\eta$, with the following property: Let $a_{0}=\left(x_{0}, t_{0}\right)$ and $a=(x, t)$ be points in $H$ with $\left|a-a_{0}\right|=s t_{0}, 0 \leq s \leq s_{0}$. Then

$$
\mu(s) \leq \frac{\left|F a-F a_{0}\right|}{\tau\left(a_{0}\right)} \leq \theta(s) .
$$

Suppose that $a_{0}, a \in H$ are as above, $\left|a-a_{0}\right|=s t_{0}, 0<s \leq 1 / 2$. Write $\tau_{0}=\tau\left(a_{0}\right), \tau=\tau(a)$. For $x, x_{0}, x_{1}, \ldots$ we write $y=f x, y_{0}=f x_{0}$, etc. Let $x_{1}$ be an arbitrary point of $S(x, t) \subset E$. Choose $x_{2} \in S\left(x_{0}, t_{0}\right)$ such that $x_{2}$ lies on the ray from $x_{0}$ through $x_{1}$. Then either

$$
\left|x_{1}-x_{2}\right|=\left|x_{2}-x_{0}\right|-\left|x_{1}-x_{0}\right| \leq t_{0}-\left|x_{1}-x\right|+\left|x-x_{0}\right| \leq t_{0}-t+\left|x-x_{0}\right|
$$

or

$$
\left|x_{1}-x_{2}\right|=\left|x_{1}-x_{0}\right|-\left|x_{2}-x_{0}\right| \leq\left|x_{1}-x\right|+\left|x-x_{0}\right|-t_{0} \leq t-t_{0}+\left|x-x_{0}\right| .
$$

Hence in both cases

$$
\left|x_{1}-x_{2}\right| \leq\left|t-t_{0}\right|+\left|x-x_{0}\right| \leq 2 s t_{0} .
$$

By quasisymmetry we obtain

$$
\begin{aligned}
\left|y_{1}-y\right| & \leq\left|y_{1}-y_{2}\right|+\left|y_{2}-y_{0}\right|+\left|y_{0}-y\right| \\
& \leq \eta\left(\frac{\left|x_{1}-x_{2}\right|}{\left|x_{0}-x_{2}\right|}\right)\left|y_{0}-y_{2}\right|+\tau_{0}+\eta\left(\frac{\left|x_{0}-x\right|}{\left|x_{0}-x_{2}\right|}\right)\left|y_{0}-y_{2}\right| \\
& \leq \eta(2 s) \tau_{0}+\tau_{0}+\eta(s) \tau_{0} \leq \tau_{0}+2 \eta(2 s) \tau_{0} .
\end{aligned}
$$

Since $x_{1} \in S(x, t)$ is arbitrary, this implies

$$
\frac{\tau-\tau_{0}}{\tau_{0}} \leq 2 \eta(2 s) \text {. }
$$

This inequality holds for all pairs $a_{0}, a$ with $\left|a-a_{0}\right|=s t_{0}$. Changing the roles of $a$ and $a_{0}$ gives

$$
\frac{\tau_{0}-\tau}{\tau} \leq 2 \eta\left(2 s t_{0} / t\right)
$$

Now $s \leq 1 / 2$ implies $\left|t-t_{0}\right| \leq t_{0} / 2$, and hence $t_{0} \leq 2 t$. From (7.29) and (7.30) we obtain

$$
\tau_{0}-\tau \leq 2 \eta(4 s)(1+2 \eta(2 s)) \tau_{0}=\theta(s) \tau_{0},
$$


where $\theta(s)=2 \eta(4 s)(1+2 \eta(2 s))$. Hence

$$
\frac{\left|\tau-\tau_{0}\right|}{\tau_{0}} \leq \theta(s)
$$

whenever $s \leq 1 / 2$.

On the other hand, since $\left|x-x_{0}\right| \leq\left|a-a_{0}\right|=s t_{0}$, we have

$$
\left|f x-f x_{0}\right| \leq \eta\left(\frac{\left|x-x_{0}\right|}{\left|x_{2}-x_{0}\right|}\right)\left|f x_{2}-f x_{0}\right| \leq \eta(s) \tau_{0} \leq \theta(s) \tau_{0} .
$$

Hence the second inequality of (7.28) is true with this $\theta$ and with $s_{0}=1 / 2$.

We turn to the first inequality of (7.28). In what follows, we let $\mu_{j}:[0, \infty) \rightarrow$ $[0, \infty)$ denote homeomorphisms depending only on $\eta$. Using the same notation as above we assume that $a_{0}, a \in H$ with $\left|a-a_{0}\right|=s t_{0}, 0<s \leq 1 / 2$. We may assume that $f$ and $f^{-1}$ are $\eta$-QS, replacing $\eta(t)$ by $\eta(t) \vee \eta^{-1}\left(t^{-1}\right)^{-1}$. Define $\mu_{1}$ and $\mu_{2}$ by

$$
\mu_{1}(s)=\frac{1}{2 \eta(2 / s)}, \quad \mu_{1}(0)=0, \quad \mu_{2}(s)=\frac{\eta^{-1}\left(\mu_{1}(s)\right)}{2} \wedge \frac{s}{4} .
$$

We consider two cases.

Case 1. $\left|x-x_{0}\right| \geq \mu_{2}(s) t_{0}$. Let $x_{4}$ be an arbitrary point of $S\left(x_{0}, t_{0}\right)$. Then

$$
\frac{\left|y_{4}-y_{0}\right|}{\left|y-y_{0}\right|} \leq \eta\left(\frac{\left|x_{4}-x_{0}\right|}{\left|x-x_{0}\right|}\right) \leq \eta\left(1 / \mu_{2}(s)\right)
$$

and hence

$$
\frac{\left|y-y_{0}\right|}{\tau_{0}} \geq \frac{1}{\eta\left(1 / \mu_{2}(s)\right)}=\mu_{3}(s)
$$

Since $\left|F a-F a_{0}\right| \geq\left|y-y_{0}\right|$, this gives the first inequality of (7.28).

Case 2. $\left|x-x_{0}\right| \leq \mu_{2}(s) t_{0}$. Since

$$
s t_{0}=\left|a-a_{0}\right| \leq\left|x-x_{0}\right|+\left|t-t_{0}\right| \leq \mu_{2}(s) t_{0}+\left|t-t_{0}\right|,
$$

one of the inequalities

$$
\begin{aligned}
& t \geq t_{0}\left(1+s-\mu_{2}(s)\right), \\
& t \leq t_{0}\left(1-s+\mu_{2}(s)\right)
\end{aligned}
$$

is true. 
Suppose first that (7.32) holds. Since $\mu_{2}(s) \leq s / 4$, we have

$$
\left|x-x_{0}\right|+t_{0} \leq \mu_{2}(s) t_{0}+t-s t_{0}+\mu_{2}(s) t_{0}<t .
$$

Hence $\bar{B}\left(x_{0}, t_{0}\right) \subset B(x, t)$. Let $x_{5} \in S\left(x_{0}, t_{0}\right)$ be arbitrary. Then $y_{5}$ and $y$ belong to $f B(x, t)$. Hence there is $y_{6} \in f S(x, t)$ such that $y_{5} \in\left[y, y_{6}\right]$. Then

$$
\frac{\left|y-y_{0}\right|}{\left|y_{5}-y_{0}\right|} \leq \eta\left(\frac{\left|x-x_{0}\right|}{\left|x_{5}-x_{0}\right|}\right) \leq \eta\left(\mu_{2}(s)\right) \leq \mu_{1}(s) \text {. }
$$

Since

$$
\left|x_{5}-x_{6}\right| \geq\left|x-x_{6}\right|-\left|x-x_{0}\right|-\left|x_{0}-x_{5}\right| \geq t-\mu_{2}(s) t_{0}-t_{0} \geq\left(s-2 \mu_{2}(s)\right) t_{0} \geq s t_{0} / 2,
$$

we have

$$
\frac{\left|y_{5}-y_{0}\right|}{\left|y_{5}-y_{6}\right|} \leq \eta\left(\frac{\left|x_{5}-x_{0}\right|}{\left|x_{5}-x_{6}\right|}\right) \leq \eta\left(\frac{2}{s}\right)=\frac{1}{2 \mu_{1}(s)}
$$

Since

$$
\tau \geq\left|y-y_{6}\right|=\left|y-y_{5}\right|+\left|y_{5}-y_{6}\right| \geq\left|y_{5}-y_{0}\right|-\left|y-y_{0}\right|+\left|y_{5}-y_{6}\right|,
$$

these inequalities give

$$
\frac{\tau-\left|y_{5}-y_{0}\right|}{\left|y_{5}-y_{0}\right|} \geq \frac{\left|y_{5}-y_{6}\right|}{\left|y_{5}-y_{0}\right|}-\frac{\left|y-y_{0}\right|}{\left|y_{5}-y_{0}\right|} \geq \mu_{1}(s) .
$$

Since $\left|y_{5}-y_{0}\right|$ is arbitrarily close to $\tau_{0}$, we obtain the desired lower bound

$$
\frac{\left|F a-F a_{0}\right|}{\tau_{0}} \geq \frac{\tau-\tau_{0}}{\tau_{0}} \geq \mu_{1}(s) .
$$

Finally assume that (7.33) is true. Since

$$
\left|x-x_{0}\right|+t \leq \mu_{2}(s) t_{0}+t_{0}\left(1-s+\mu_{2}(s)\right) \leq t_{0}(1-s+s / 2)<t_{0},
$$

we have $\bar{B}(x, t) \subset B\left(x_{0}, t_{0}\right)$. Let $x_{7} \in S(x, t)$ be arbitrary. Since $y_{0}, y_{7} \in$ $f B\left(x_{0}, t_{0}\right)$, there is $y_{8} \in f S\left(x_{0}, t_{0}\right)$ such that $y_{7} \in\left[y_{0}, y_{8}\right]$. Since

$$
t \geq t_{0}-\left|t-t_{0}\right| \geq t_{0}-s t_{0} \geq t_{0} / 2
$$

we have

$$
\frac{\left|y-y_{0}\right|}{\left|y-y_{7}\right|} \leq \eta\left(\frac{\left|x-x_{0}\right|}{\left|x-x_{7}\right|}\right) \leq \eta\left(\frac{\mu_{2}(s) t_{0}}{t}\right) \leq \eta\left(2 \mu_{2}(s)\right) \leq \mu_{1}(s) .
$$


By (7.33) and the inequality $\mu_{2}(s) \leq s / 4$ we have $t \leq(1-3 s / 4) t_{0}<t_{0}$. Hence

$$
\left|x_{8}-x_{7}\right| \geq\left|x_{8}-x_{0}\right|-\left|x_{0}-x\right|-\left|x-x_{7}\right| \geq t_{0}-\mu_{2}(s) t_{0}-t \geq s t_{0} / 2>s t / 2 .
$$

Consequently,

$$
\frac{\left|y-y_{7}\right|}{\left|y_{8}-y_{7}\right|} \leq \eta\left(\frac{\left|x-x_{7}\right|}{\left|x_{8}-x_{7}\right|}\right) \leq \eta\left(\frac{2}{s}\right)=\frac{1}{2 \mu_{1}(s)}
$$

Since

$$
\tau_{0} \geq\left|y_{8}-y_{0}\right|=\left|y_{7}-y_{0}\right|+\left|y_{8}-y_{7}\right| \geq\left|y_{7}-y\right|-\left|y-y_{0}\right|+\left|y_{8}-y_{7}\right|,
$$

these inequalities yield

$$
\frac{\tau_{0}-\left|y_{7}-y\right|}{\left|y_{7}-y\right|} \geq \frac{\left|y_{8}-y_{7}\right|}{\left|y_{7}-y\right|}-\frac{\left|y-y_{0}\right|}{\left|y_{7}-y\right|} \geq \mu_{1}(s)
$$

and hence

$$
\frac{\tau_{0}-\tau}{\tau} \geq \mu_{1}(s) .
$$

Choose $s_{0} \in(0,1 / 2]$ such that $\theta\left(s_{0}\right) \leq 1 / 2$. If $s \leq s_{0}$, then (7.31) implies that $\tau \geq \tau_{0} / 2$. Hence

$$
\frac{\left|F a-F a_{0}\right|}{\tau_{0}} \geq \frac{\tau_{0}-\tau}{2 \tau} \geq \mu_{1}(s) / 2 .
$$

We have now proved (7.28) for all $s \leq s_{0}$.

From (7.28) it follows that $F$ is continuous in $H$. If $\left|x-x_{0}\right| \leq t$, then $\tau(x, t) \leq \tau\left(x_{0}, 2 t\right)$. Hence $\bar{F}$ is continuous in $\bar{H}$. Furthermore, (7.28) implies that $\bar{F}$ maps each ray $\left\{x_{0}\right\} \times[0, \infty)$ homeomorphically onto the ray $\left\{f x_{0}\right\} \times[0, \infty)$. From Lemma 7.34 below it follows that $\bar{F}$ is a homeomorphism.

By (7.28), the homeomorphism $F: H \rightarrow H^{\prime}$ is $\left(\theta, s_{0}\right)$-relative in the sense of I.3.6. If $a_{0}, a \in H$ and $\left|a-a_{0}\right|=s t_{0}$ with $s \leq s_{0}$, then

$$
\left|F a-F a_{0}\right| \geq \mu(s) \tau_{0} .
$$

Since $F$ is a homeomorphism, this implies that $F B\left(a_{0}, s t_{0}\right)$ contains the ball $B\left(F a_{0}, \mu(s) \tau_{0}\right)$. Hence $F^{-1}$ is $\left(\theta_{1}, s_{1}\right)$-relative with $s_{1}=\mu\left(s_{0}\right), \theta_{1}=\mu^{-1}$. From I.3.8 it follows that $F$ is $\varphi$-solid with $\varphi=\varphi_{\eta}$. $\square$

7.34. Lemma. Suppose that $X$ and $Y$ are topological spaces and that $F: X \times[0, \infty) \rightarrow Y \times[0, \infty)$ is a continuous bijective map of the form $F(x, t)=$ $(f x, \tau(x, t))$ where $f: X \rightarrow Y$ is a homeomorphism. Then $F$ is a homeomorphism. 
Proof. Let $a_{0}=\left(x_{0}, t_{0}\right) \in X \times[0, \infty)$. We must show that $F$ is open at $x_{0}$. Suppose first that $t_{0}>0$. Let $W=U \times V$ be a neighborhood of $a_{0}$, where $U$ is open in $X$ and $t_{0} \in V=\left(t_{1}, t_{2}\right) \subset[0, \infty)$. The map $t \mapsto \tau\left(x_{0}, t\right)$ is a self homeomorphism of $[0, \infty)$. Choose numbers $u_{1}, u_{2}$ with

$$
\tau\left(x_{0}, t_{1}\right)<u_{1}<\tau\left(x_{0}, t_{0}\right)<u_{2}<\tau\left(x_{0}, t_{2}\right) .
$$

Since $\tau$ is continuous, there is a neighborhood $U_{1} \subset U$ of $x_{0}$ such that $x \in U_{1}$ implies $\tau\left(x, t_{1}\right)<u_{1}$ and $\tau\left(x, t_{2}\right)>u_{2}$. Since $F$ maps the vertical segment $\{x\} \times$ $\left(t_{1}, t_{2}\right)$ onto $\{f x\} \times\left(\tau\left(x, t_{1}\right), \tau\left(x, t_{2}\right)\right)$, the set $F W$ contains the neighborhood $f U_{1} \times\left(u_{1}, u_{2}\right)$ of $F a_{0}$.

The case $t_{0}=0$ is proved by an obvious modification of the argument above.

7.35. Reflection. The reflection principle in the QS theory of $R^{n}$ enables us to extend a $K$-QC map between balls or half spaces to a $K$-QC map of the whole space $\dot{R}^{n}$. We shall next present a free version of this idea. In fact, it turns out that the principle follows easily from a general result on unions of QS maps.

Suppose first that $G$ and $G^{\prime}$ are unit balls of $E$ and $E^{\prime}$, respectively, and that $f: G \rightarrow G^{\prime}$ is a $\varphi$-FQC map with $f(0)=0$. Then $f$ is $\eta$-QM with $\eta=\eta(\varphi)$ by 6.5 and 7.16. In particular, $f$ extends to a homeomorphism $\bar{f}: \bar{G} \rightarrow \bar{G}^{\prime}$. Let $u$ and $u^{\prime}$ be the inversions $x \mapsto x /|x|^{2}$ of $\dot{E}$ and $\dot{E}^{\prime}$, respectively. Then we can extend $\bar{f}$ to a homeomorphism $f^{*}: E \rightarrow \dot{E}^{\prime}$ by setting $f^{*} x=u^{\prime} f u x$ for $x \in \dot{E} \backslash \bar{G}$. We say that $f^{*}$ is obtained from $f$ by reflection.

Next assume that $G$ is a half space in $E$ with $0 \in \partial G$. This means that $\partial G \backslash\{\infty\}$ is a closed linear subspace $T$ of $E$ of codimension 1 . Let $e \in G$ be a unit vector. Then $E$ is spanned by $T \cup\{e\}$, and there is a unique linear map $v: E \rightarrow E$ such that $v \mid T=\mathrm{id}$ and $v e=-e$. We say that $v$ is a reflection in $T$. If $E$ is Hilbert space, we can choose $e$ to be orthogonal to $T$, and then $v$ is an isometry. In a Banach space, an isometric reflection does not always exist, but for every $M>3$, there is an $M$-bilipschitz reflection; see Lemma 7.37 below. In what follows, we assume that the reflections are 4-bilipschitz.

Suppose that $G^{\prime}$ is another half space with $0 \in T^{\prime}=\partial G^{\prime} \backslash\{\infty\}$. Let $f: G \rightarrow$ $G^{\prime}$ be $\varphi$-FQC. Again by uniformity, $f$ is $\eta$-QM, $\eta=\eta(\varphi)$, and extends to a homeomorphism $\bar{f}: \bar{G} \rightarrow \bar{G}^{\prime}$. Suppose that $\bar{f}(\infty)=\infty$. Using reflections $v$ and $v^{\prime}$ in $T$ and in $T^{\prime}$, respectively, we extend $\bar{f}$ to a homeomorphism $f^{*}: \dot{E} \rightarrow \dot{E}^{\prime}$ with $f^{*} x=v^{\prime} f v x$ for $x \in E \backslash \bar{G}$. We again say that $f^{*}$ is obtained from $f$ by reflection.

In both cases we have $f^{*}(\infty)=\infty$. Hence $f^{*}$ defines a homeomorphism $f_{*}: E \rightarrow E^{\prime}$. We want to show that this map is $\varphi_{1}$-FQC with $\varphi_{1}=\varphi_{1}(\varphi)$. Since the inversions $u, u^{\prime}$ and the reflections $v, v^{\prime}$ are $\eta_{0}$-QM with a universal $\eta_{0}$, the maps $f^{*} \mid \bar{G}$ and $f^{*} \mid E \backslash G$ are $\eta_{1}$-QM with $\eta_{1}=\eta_{1}(\varphi)$. Since $f^{*}(\infty)=\infty$, $f^{*} \mid \bar{G} \backslash\{\infty\}$ is $\eta_{1}$-QS in the second case, and $f^{*} \mid E \backslash G$ is $\eta_{1}$-QS in both cases. 
From Lemma 7.38 below it follows that in the first case, $f_{*} \mid \bar{G}$ is $\eta_{2}$-QS with $\eta_{2}=\eta_{2}(\varphi)$. From a general result of QS theory, given as Theorem 7.39 below, it follows that $f_{*}$ is $\eta_{3}$-QS with $\eta_{3}=\eta_{3}(\varphi)$. This implies that $f_{*}$ is $\varphi_{1}$-FQC with $\varphi_{1}=\varphi_{1}(\varphi)$ by I.5.18, and we obtain the following result:

7.36. Theorem. (Reflection principle). Suppose that $f_{*}: E \rightarrow E^{\prime}$ is obtained from a $\varphi$-FQC map $f: G \rightarrow G^{\prime}$ by reflection. Then $f_{*}$ is $\varphi_{1}-\mathrm{FQC}$ with $\varphi_{1}=\varphi_{1}(\varphi)$.

7.37. Lemma. Suppose that $H \subset E$ is a half space and that $M>3$. Then there is an $M$-bilipschitz reflection of $E$ in $\partial H$.

Proof. Setting $r=2 /(M-1)$ we have $0<r<1$. Fix an arbitrary $h_{0} \in H$. Write $T=\partial H$ and choose $a \in T$ such that $\left|h_{0}-a\right|<d\left(h_{0}, T\right) / r$. Write $h_{1}=$ $h_{0}-a$ and $e=h_{1} /\left|h_{1}\right|$. Then $e \in H,|e|=1$, and

$$
d(e, T)=d\left(h_{1}, T\right) /\left|h_{1}\right|=d\left(h_{0}, T\right) /\left|h_{1}\right|>r .
$$

Let $v$ be the reflection in $T$ with $v e=-e$. We show that the norm $|v|$ of $v$ is at most $M$.

Let $x \in E$ be a unit vector. We can write $x=y+t e$ with $y \in T, t \in R^{1}$. Then

$$
1=|x| \geq d(x, T)=d(t e, T)=|t| d(e, T) \geq|t| r .
$$

Hence

$$
|v x|=|x-2 t e| \leq|x|+2|t| \leq 1+2 / r=M,
$$

which implies that $|v| \leq M$. Since $v^{-1}=v$, the map $v$ is $M$-bilipschitz. $\square$

7.38. Lemma. Suppose that $G$ and $G^{\prime}$ are the unit balls of $E$ and $E^{\prime}$, respectively, and that $f: \bar{G} \rightarrow \bar{G}^{\prime}$ is an $\eta$-QM homeomorphism with $f(0)=0$ and $f \partial G=\partial G^{\prime}$. Then $f$ is $\eta_{1}-\mathrm{QS}$ with $\eta_{1}=\eta_{1}(\eta)$.

Proof. Fix $z_{1} \in \partial G$ and set $z_{2}=-z_{1}$ and $z_{3}=0$. By [Vä $\left.\ddot{a}_{1}, 3.12\right]$ or by 5.9 , it suffices to show that $\left|f z_{1}-f z_{2}\right| \geq \lambda$ for some $\lambda=\lambda(\eta)>0$. Setting $z_{\mathbf{4}}=f^{-1}\left(-f z_{1}\right)$ we have

$$
\frac{\left|z_{1}-z_{4}\right|\left|z_{2}-0\right|}{\left|z_{1}-z_{2}\right|\left|z_{4}-0\right|} \leq 1, \quad \frac{\left|f z_{1}-f z_{4}\right|\left|f z_{2}-0\right|}{\left|f z_{1}-f z_{2}\right|\left|f z_{4}-0\right|}=\frac{2}{\left|f z_{1}-f z_{2}\right|}
$$

and hence $\left|f z_{1}-f z_{2}\right| \geq 2 / \eta(1)$.

7.39. Theorem. Suppose that $f: E \rightarrow E^{\prime}$ is a homeomorphism and that $E=A_{1} \cup A_{2}$ such that $f \mid A_{1}$ and $f \mid A_{2}$ are $\eta$-QS. Then $f$ is $\eta_{1}$-QS with $\eta_{1}=\eta_{1}(\eta)$. 
Proof. Replacing the sets $A_{1}, A_{2}$ with their closures, we may assume that they are closed in $E$. By I.5.5, it suffices to show that $f$ is weakly $H$-QS with $H=H(\eta)$. Suppose that $x, a, b$ are distinct points in $E$ with $|a-x|=|b-x|$. In the present situation, it suffices to show that $|f a-f x| \leq H(\eta)|f b-f x|$. We may normalize the situation so that $x=0, f x=0$, and $|a|=|b|=1=|f b|$. Then we must find an upper bound $|f a| \leq H(\eta)$. We may assume that $0 \in A_{1}$ and that $\{a, b\} \not \subset A_{1}$. We consider three cases.

Case 1. $a \in A_{1}, b \in A_{2}$. Choose $x_{1} \in A_{1} \cap A_{2}$ with $f x_{1} \in[0, f b]$. If $\left|x_{1}\right| \geq 1 / 2$, we have

$$
|f a| \leq \eta\left(\frac{|a|}{\left|x_{1}\right|}\right)\left|f x_{1}\right| \leq \eta(2) .
$$

Suppose that $\left|x_{1}\right| \leq 1 / 2$. Since $S(1)$ is connected, there is $x_{2} \in S(1) \cap A_{1} \cap A_{2}$. Then

$$
\left|f x_{2}-f x_{1}\right| \leq \frac{\left|f x_{2}-f x_{1}\right|}{\left|f b-f x_{1}\right|} \leq \eta\left(\frac{\left|x_{2}-x_{1}\right|}{\left|b-x_{1}\right|}\right) \leq \eta(3),
$$

and hence

$$
|f a| \leq \eta\left(\frac{|a|}{\left|x_{2}\right|}\right)\left|f x_{2}\right| \leq \eta(1)(1+\eta(3)) .
$$

Case 2. $a \in A_{2}, b \in A_{1}$. Choose $x_{2} \in S(1) \cap A_{1} \cap A_{2}$ as above and a point $x_{3} \in[0, a] \cap A_{1} \cap A_{2}$. Then

$$
\begin{aligned}
\left|f a-f x_{3}\right| & \leq \eta\left(\frac{\left|a-x_{3}\right|}{\left|x_{2}-x_{3}\right|}\right) \eta\left(\frac{\left|x_{2}-x_{3}\right|}{\left|x_{2}\right|}\right) \eta\left(\frac{\left|x_{2}\right|}{|b|}\right)|f b| \leq \eta(1) \eta(2) \eta(1), \\
\left|f x_{3}\right| & \leq \eta\left(\frac{\left|x_{3}\right|}{|b|}\right)|f b| \leq \eta(1),
\end{aligned}
$$

and hence $|f a| \leq \eta(1)+\eta(1)^{2} \eta(2)$.

Case 3. $a \in A_{2}, b \in A_{2}$. We again choose $x_{1} \in A_{1} \cap A_{2}$ with $f x_{1} \in[0, f b]$. If $\left|x_{1}\right| \leq 1 / 2$, then

$$
\left|f a-f x_{1}\right| \leq \eta\left(\frac{\left|a-x_{1}\right|}{\left|b-x_{1}\right|}\right)\left|f b-f x_{1}\right| \leq \eta(3),
$$

and hence $|f a| \leq 1+\eta(3)$. Suppose that $\left|x_{1}\right| \geq 1 / 2$. Choose again $x_{3} \in[0, a] \cap$ $A_{1} \cap A_{2}$. Then

$$
\begin{aligned}
\left|f x_{3}\right| & \leq \eta\left(\frac{\left|x_{3}\right|}{\left|x_{1}\right|}\right)\left|f x_{1}\right| \leq \eta(2), \\
\left|f a-f x_{3}\right| & \leq \eta\left(\frac{\left|a-x_{3}\right|}{\left|b-x_{3}\right|}\right)\left|f b-f x_{3}\right| \leq \eta(1)\left(1+\left|f x_{3}\right|\right),
\end{aligned}
$$

and hence $|f a| \leq \eta(2)+\eta(1)(1+\eta(2))$. व 
7.40. Remark. The one-dimensional version of 7.39 is false. For example, define $f: R^{1} \rightarrow R^{1}$ by $f x=x$ for $x \leq 0$ and $f x=x^{2}$ for $x \geq 0$. Then $f$ is not QS although it is QS in $(-\infty, 0]$ and in $[0, \infty)$.

7.41. Hyperbolic geometry. Suppose that $E$ is a Hilbert space and that $G$ is a half space of $E$. Then the QH metric $k$ of $G$ is the hyperbolic metric of $G$. Each pair $a, b \in G$ can be joined by a unique hyperbolic segment, which is a subarc of a semicircle or a ray which joins two points of $\partial G$ and is orthogonal to $\partial G$. This semicircle or ray is called a hyperbolic line. The Möbius maps preserving $G$ are the hyperbolic isometries of $G$.

Maps related to $\mathrm{CQH}$ maps of $G$ have been studied by several people. For example, Thurston [Th, 5.9] considers maps $f: G \rightarrow G$ called pseudo-isometries. They are defined by the condition

$$
(k(x, y)-C) / M \leq k(f x, f y) \leq M k(x, y) .
$$

Essential use has been made by the fact that the image of a hyperbolic line lies in a hyperbolic neighborhood of another hyperbolic line. We next show that for CQH maps, this result follows easily from the fact (Theorem 7.9) that $f$ extends to a map which is QM rel $\partial G$. However, I feel that in the applications it is usually easier to make direct use of Theorem 7.9 or the related results 7.14 and 7.15. Moreover, these results also apply to many other domains, in which we do not have well-defined hyperbolic lines.

The hyperbolic metric $h$ of the unit ball $B(1)$ is obtained with the aid of any Möbius map of $G$ onto $B(1)$. Alternatively, $h$ is defined by the density $2 /\left(1-|x|^{2}\right)$. Then $h$ and the QH metric $k$ of $B(1)$ satisfy the inequalities $k \leq h \leq 2 k$.

7.42. Theorem. Suppose that $G$ is a half space of a Hilbert space and that $f: G \rightarrow G$ is $(M, C)$-CQH. Let $\gamma$ be a hyperbolic line in $G$. Then there is a unique hyperbolic line $\gamma^{\prime}$ such that $f \gamma$ lies in the hyperbolic neighborhood

$$
N\left(\gamma^{\prime}, r\right)=\left\{y \in G: k\left(y, \gamma^{\prime}\right) \leq r\right\},
$$

where $r$ can be chosen to depend only on $(M, C)$.

Proof. Let $a$ and $b$ be the endpoints of $\gamma$. By 7.9, $f$ extends to a homeomorphism $\bar{f}: \bar{G} \rightarrow \bar{G}$. By auxiliary Möbius maps we may assume that $a=0=\bar{f} a$ and $b=\infty=\bar{f} b$. Then $\gamma$ is the ray from 0 to $\infty$, orthogonal to $\partial G$. The uniqueness of $\gamma^{\prime}$ is clear: we must have $\gamma^{\prime}=\gamma$. The neighborhood $N(\gamma, r)$ is a cone with axis $\gamma$. Let $x \in \gamma$, and let $y \in \partial G$ be the point for which $\bar{f} y$ is the orthogonal projection of $f x$ on $\partial G$. Let $\alpha$ be the angle between the vector $f x$ and the ray $\gamma$. It suffices to obtain an upper bound for $\tan \alpha$ in terms of $(M, C)$. 
By $7.9, \bar{f}$ is $\eta$-QM rel $\partial G$ with $\eta$ depending only on $(M, C)$. Applied to the quadruple $(y, 0, x, \infty)$ this gives

$$
\tan \alpha=\frac{|\bar{f} y|}{|\bar{f} y-f x|} \leq \eta\left(\frac{|y|}{|y-x|}\right) \leq \eta(1) .
$$

This proves the theorem. From the formulas in $\left[\mathrm{Vu}_{2}\right.$, p. 22] we can obtain the explicit bound $r=\ln \left(H+\sqrt{1+H^{2}}\right)$ with $H=\eta(1)$. व

\section{Distortion}

8.1. Introduction to Section 8. This section was inspired by a manuscript [Co] of D. Cooper. Let $B^{n}$ be the open unit ball of $R^{n}$, and let $f: B^{n} \rightarrow B^{n}$ be $(M, C)$-CQH. Cooper considered the distortion of hyperbolic spheres under $f$. By auxiliary Möbius homeomorphisms of $B^{n}$ we reduce the problem to the case where $f(0)=0$ and the center of the sphere is the origin. We can then consider the $\mathrm{QH}$ sphere

$$
S_{k}(r)=\partial B_{k}(r)=\left\{x \in B^{n}: k(x, 0)=r\right\},
$$

where $k$ is the $\mathrm{QH}$ metric of $B^{n}$. This sphere is also a euclidean sphere $S(s)$ with radius $s=1-e^{-r}$. If $r \geq 2 C$, then

$$
r / 2 M \leq k(f x, 0) \leq 2 M r
$$

for all $x \in S_{k}(r)$. Examples show that for any $r>0, k(f x, 0)$ may have the order of magnitude of $M r$ or $r / M$ at certain points of $S_{k}(r)$. Cooper made the important new observation that for large $r$, this can happen only in a set of small area, provided that $n \geq 3$. Indeed, the multiplicative bounds in (8.2) can be replaced by the additive bounds

$$
r-\alpha \leq k(f x, 0) \leq r+\alpha
$$

for $x \in S_{k}(r)$ except for a subset whose area has an upper bound $\varepsilon(\alpha, M, C, n)$ which tends to zero as $\alpha \rightarrow \infty$. Observe that $\varepsilon$ does not depend on $r$.

I have not been able to follow all details of Cooper's proof. The purpose of this section is to give a new proof for this result. In fact, we prove in Theorem 8.7 that the first inequality of $(8.3)$ also holds for $n=2$. To prove the second inequality we need the absolute continuity of the boundary map $S^{n-1} \rightarrow S^{n-1}$. This requires $n \geq 3$; the result is given as Theorem 8.9.

Since we are working in $R^{n}$, this section differs from the preceding sections. However, our proof is based on Theorem 7.15 on relative quasisymmetry. On the other hand, the result is also new for $\mathrm{QC}$ maps of $B^{n}$. A reader interested only in this special case can skip the preceding sections, since a $K$-QC map $f: B^{n} \rightarrow B^{n}$ with $f(0)=0$ is well known to be $\eta$-QS with $\eta=\eta_{K}$. For example, reflect $f$ to $R^{n}$ and use [AVV, 5.23]. 
8.4. Notation. Suppose that $f: X \rightarrow Y$ is a map. For $x \in X$ and $r>0$ we set $S(x, r)=\partial B(x, r)$ and

$$
\begin{aligned}
L(x, f, r) & =\sup \{|f y-f x|: y \in S(x, r)\} \\
l(x, f, r) & =\inf \{|f y-f x|: y \in S(x, r)\} .
\end{aligned}
$$

The $n$-dimensional outer measure of a set $A \subset S^{n}=\partial B^{n+1}$ is written as $m_{n}(A)$. For $x \in S^{n}, 0<r \leq 2$, we let $U(x, r)$ be the cap $S^{n} \cap B(x, r)$. There are positive numbers $a_{n}<b_{n}$ such that

$$
a_{n} r^{n} \leq m_{n}(U(x, r)) \leq b_{n} r^{n}
$$

for all $x \in S^{n}$ and $0<r \leq 2$. We also write $\omega_{n}=m_{n}\left(S^{n}\right)$.

8.5. Lemma. Suppose that $n \geq 1$ and that $f: S^{n} \rightarrow S^{n}$ is a homeomorphism. Suppose also that $0<r \leq 2$, that $t>0$ and that $A \subset S^{n}$ is such that

$$
l(x, f, r) \geq t r
$$

for all $x \in A$. Then $m_{n}(A) \leq \mu(t, n)$ where $\mu(t, n) \rightarrow 0$ as $t \rightarrow \infty$.

Proof. The proof is based on a simple packing argument. Choose a maximal set $F \subset A$ with the property that $|x-y| \geq 2 r$ whenever $x, y \in F$ with $x \neq y$. Write $k=\operatorname{card} F$. Since $A$ is covered by the caps $U(a, 2 r), a \in F$, we have $m_{n}(A) \leq k b_{n}(2 r)^{n}$. Hence it suffices to find an estimate

$$
k r^{n} \leq \mu(t, n) .
$$

Since

$$
f U(a, r) \supset U(f a, l(a, f, r)) \supset U(f a, t r)
$$

for $a \in F$ and since the sets $f U(a, r)$ are disjoint, we have

$$
\omega_{n}=m_{n}\left(S^{n}\right) \geq \sum_{a \in F} m_{n}(f U(a, r)) \geq k a_{n}(t r)^{n} .
$$

This gives (8.6) with $\mu(t, n)=\omega_{n} / a_{n} t^{n}$. व

8.7. Theorem. Suppose that $n \geq 2$, that $f: B^{n} \rightarrow B^{n}$ is $(M, C)-\mathrm{CQH}$, that $f(0)=0$, and that $0<\alpha<r$. Suppose also that $A$ is a subset of the QH sphere $S_{k}(r)$ such that $k(f x, 0) \leq r-\alpha$ for all $x \in A$. Then

$$
m_{n-1}(A) \leq \varepsilon_{1}(\alpha, M, C, n)
$$

where $\varepsilon_{1}(\alpha, M, C, n) \rightarrow 0$ as $\alpha \rightarrow \infty$. 
Proof. By 7.15, $f$ extends to a homeomorphism $\bar{f}: \bar{B}^{n} \rightarrow \bar{B}^{n}$ which is $\eta$-QS rel $S^{n-1}$ with $\eta=\eta_{M, C}$. Let $f_{0}: S^{n-1} \rightarrow S^{n-1}$ be the restriction of $\bar{f}$. Writing as usual $\delta(x)=d\left(x, \partial B^{n}\right)=1-|x|$ we have $\delta(a)=e^{-r}$ and $\delta(f a) \geq e^{\alpha-r}$ for all $a \in A$. Let $p: R^{n} \backslash\{0\} \rightarrow S^{n-1}$ be the radial projection $p x=x /|x|$. We first show that

$$
l\left(p a, f_{0}, e^{-r}\right) \geq e^{\alpha-r} / \eta(1)
$$

for each $a \in A$. Let $y \in S^{n-1}$ with $|y-p a|=e^{-r}$. Then $|a-p a|=|y-p a|$, and hence

$$
e^{\alpha-r} \leq \delta(f a) \leq\left|f a-f_{0} p a\right| \leq \eta(1)\left|f_{0} y-f_{0} p a\right| .
$$

This proves (8.8). By Lemma 8.5 we obtain

$$
m_{n-1}(A) \leq m_{n-1}(p A) \leq \mu\left(e^{\alpha} / \eta(1), n-1\right)=\varepsilon_{1}(\alpha, M, C, n) \text {. } \square
$$

8.9. Theorem. Suppose that $n \geq 3$, that $f: B^{n} \rightarrow B^{n}$ is $(M, C)-\mathrm{CQH}$, that $f(0)=0$ and that $r>0, \alpha>0$. Suppose also that $A$ is a subset of the QH sphere $S_{k}(r)$ such that $k(f x, 0) \geq r+\alpha$ for all $x \in A$. Then

$$
m_{n-1}(A) \leq \varepsilon_{2}(\alpha, M, C, n)
$$

where $\varepsilon_{2}(\alpha, M, C, n) \rightarrow 0$ as $\alpha \rightarrow \infty$.

Proof. Let $a \in A$. Using the notation of the proof of 8.7 we first show that

$$
L\left(p a, f_{0}, e^{-r}\right) \leq c e^{-r-\alpha}
$$

with $c=c(M, C)$. Assume that $y \in S^{n-1}$ with $|y-p a|=e^{-r}$. Set $z=f_{0}^{-1} p f a$. Since

$$
|z-p a| \leq|z-a|+|a-p a| \leq 2|z-a|
$$

the relative $\eta$-quasisymmetry of $\bar{f}$ gives

$$
\left|f_{0} z-f_{0} p a\right| \leq \eta(2)\left|f_{0} z-f a\right| .
$$

This implies

$$
\left|f a-f_{0} p a\right| \leq(1+\eta(2))\left|f_{0} z-f a\right| \leq(1+\eta(2)) e^{-r-\alpha} .
$$

Since $|y-p a|=|a-p a|$, we have

$$
\left|f_{0} y-f_{0} p a\right| \leq \eta(1)\left|f a-f_{0} p a\right| .
$$

These estimates yield (8.10) with $c=\eta(1)(1+\eta(2))$.

From (8.10) it follows that

$$
l\left(b, f_{0}^{-1}, c e^{-r-\alpha}\right) \geq e^{-r}
$$

for each $b \in f_{0} p A$. Hence Lemma 8.5 gives

$$
m_{n-1}\left(f_{0} p A\right) \leq \mu\left(e^{\alpha} / c, n-1\right)=\varepsilon_{3}(\alpha, M, C, n),
$$

and $\varepsilon_{3} \rightarrow 0$ as $\alpha \rightarrow \infty$. The theorem now follows easily from the absolute continuity of $\mathrm{QC}$ maps. This is the point where the condition $n \geq 3$ is needed. We give the required result as Lemma 8.11 below. $\square$ 
8.11. Lemma. Suppose that $n \geq 2$ and that $f: S^{n} \rightarrow S^{n}$ is an $\eta$-QS homeomorphism. Then for each $A \subset S^{n}$ we have $m_{n}(f A) \leq \varepsilon\left(m_{n}(A), \eta, n\right)$, where $\varepsilon(t, \eta, n) \rightarrow 0$ as $t \rightarrow 0$.

Proof. Let $\varphi: S^{n} \rightarrow \dot{R}^{n}$ be the stereographic projection with $\varphi\left(e_{n+1}\right)=\infty$. Then $\varphi$ maps the lower hemisphere $H$ onto $B^{n}$. Let $Q \subset \bar{B}^{n}$ be a closed $n$-cube with vertices on $S^{n-1}$. Since there is an integer $k=k(n)$ such that $S^{n}$ can be covered by $k$ rotations of $\varphi^{-1} Q$, we may assume that $A \subset \varphi^{-1} Q$. We may also assume that $f e_{n+1}=e_{n+1}$. The map $g=\varphi f \varphi^{-1}: \dot{R}^{n} \rightarrow \dot{R}^{n}$ is a $K$-QC map with $g(\infty)=\infty$ and $K=\eta(1)^{n-1}$. From the $\eta$-quasisymmetry of $f$ we obtain a lower bound $\left|f x-e_{n+1}\right| \geq q=q(\eta)>0$ for each $x \in H$. Indeed, setting $y=f^{-1}\left(-e_{n+1}\right)$ we have

$$
\left|y-e_{n+1}\right| \leq 2 \leq \sqrt{2}\left|x-e_{n+1}\right|
$$

and hence

$$
2=\left|f y-f e_{n+1}\right| \leq \eta(\sqrt{2})\left|f x-e_{n+1}\right| .
$$

It follows that $m(g Q)$ has an upper bound depending only on $\eta$ and $n$. From [Re, Corollary, p. 262] we obtain an estimate

$$
m(g \varphi A) \leq \varepsilon_{0}(m(\varphi A), \eta, n)
$$

where $\varepsilon_{0}(t, \eta, n) \rightarrow 0$ as $t \rightarrow 0$. This proves the lemma, since $\varphi$ is $L$-bilipschitz in $S^{n} \backslash B\left(e_{n+1}, q\right)$ with $L=L(\eta, n)$. व

\section{References}

[Al] Alestalo, P.J.: Kvasisymmetria tuloavaruuksissa ja uniformiset alueet. - Licentiate's thesis, University of Helsinki, 1991 (unpublished).

[AVV] Anderson, G.D., M.K. Vamanamurthy and M. Vuorinen: Dimension-free quasiconformal distortion in $n$-space. - Trans. Amer. Math. Soc. 297, 1986, 687-706.

[Co] Cooper, D.: Quasi-isometries of hyperbolic space are almost isometries. - Manuscript, 1988.

[DS] DE-Spiller, D.A.: Equimorphisms and quasi-conformal mappings of the absolute. - Soviet Math. Dokl. 11, 1970, 1324-1328.

[ET] Efremovich, V.A., and E.S. Tihomirova: Equimorphisms of hyperbolic spaces. - Izv. Akad. Nauk SSSR 28, 1964, 1139-1144 (Russian).

[Ge] Gehring, F.W.: Uniform domains and the ubiquitous quasidisk. - Jahresber. Deutsch. Math.-Verein. 89, 1987, 88-103.

[GO] Gehring, F.W., and B.G. Osgood: Uniform domains and the quasi-hyperbolic metric. - J. Analyse Math. 36, 1979, 50-74.

[Gr] Gromov, M.: Hyperbolic manifolds, groups and actions. - Riemann surfaces and related topics, Ann. of Math. Stud. 97, Princeton University Press, 1981, 183-213.

[Jo] Jones, P.W.: Extension theorems for BMO. - Indiana Univ. Math. J. 29, 1980, 41-66. 
[Ma] Martio, O.: Definitions for uniform domains. - Ann. Acad. Sci. Fenn. Ser. A I Math. 5, 1980, 197-205.

[MS] Martio, O., and J. Sarvas: Injectivity theorems in plane and space. - Ann. Acad. Sci. Fenn. Ser. A I Math. 4, 1979, 383-401.

[Re] ReimanN, H.M.: Functions of bounded mean oscillation and quasiconformal mappings. Comment. Math. Helv. 49, 1974, 260-276.

[Sc] SchäfFER, J.J.: Inner diameter, perimeter, and girth of spheres. - Math. Ann. 173, 1967, 59-82.

[Th] Thurston, W.P.: The geometry and topology of three-manifolds. - Mimeographed notes, Princeton University, 1980.

[TV 1 ] TUKIA, P., and J. VÄISÄLÄ: Quasisymmetric embeddings of metric spaces. - Ann. Acad. Sci. Fenn. Ser. A I Math. 5, 1980, 97-114.

[TV $\left.{ }_{2}\right]$ Tukia, P., and J. VÄIsÄLÄ: Quasiconformal extension from dimension $n$ to $n+1$. - Ann. of Math. 115, 1982, 331-348.

[TV 3 ] TukIA, P., and J. VÄISÄLÄ: Lipschitz and quasiconformal approximation and extension. - Ann. Acad. Sci. Fenn. Ser. A I Math. 6, 1981, 303-342.

[Vä ${ }_{1}$ ] VÄISÄLÄ, J.: Quasimöbius maps. - J. Analyse Math. 44, 1985, 218-234.

[Vä] ] VÄIsÄLÄ, J.: Uniform domains. - Tôhoku Math. J. 40, 1988, 101-118.

[Vä ${ }_{3}$ ] VÄIsÄLÄ, J.: Quasiconformal concordance. - Monatsh. Math. 107, 1989, 155-168.

[Vä4] VÄIs ̈̈LÄ, J.: Free quasiconformality in Banach spaces I. - Ann. Acad. Sci. Fenn. Ser. A I Math. 15, 1990, 355-379.

[Vä ${ }_{5}$ ] V̈̈ISÄL̈̈, J.: Free quasiconformality in Banach spaces III. - In preparation.

[Vu ${ }_{1}$ ] Vuorinen, M.: Conformal invariants and quasiregular mappings. - J. Analyse Math. 45, $1985,69-115$.

[Vu $\left.{ }_{2}\right]$ Vuorinen, M.: Conformal geometry and quasiregular mappings. - Lecture Notes in Mathematics 1319, Springer-Verlag, 1988.

Helsingin yliopisto

Matematiikan laitos

Hallituskatu 15

SF-00100 Helsinki

Finland

Received 16 October 1990 